\title{
EPIDEMIOLOGICAL HIGHLIGHTS ON SOME VIRUSES ASSOCIATED WITH INCREASED MORTALITY IN CHICKENS BRED IN SHARKIA GOVERNORATE.
}

\author{
BAYOUMIE, H.A.A.* and HASANEEN, O.A.** \\ ${ }^{*}$ Senior Researcher, Animal health Res. Inst. Zagazig (Poult. Dis. Dept.) \\ ${ }^{* * *}$ Lecture of Poult. Dis. (Dept. of Poult. and Rabbit Dis. Faculty. Vet. Med. Zagazig Univ.) \\ Email: heshambayoumie@yahoo.com $\quad$ Assiut University Email: $\underline{\text { www.aun.edu.eg }}$
}

\section{ABSTRACT}

Received at: 21/3/2015

Accepted: 20/4/2015
In the present study 1317 serum samples obtained from 6 broiler flocks, 2 broiler breeders and 28 commercial layer flocks were examined for $\mathrm{HI}$ antibodies of \{NDV, IBV (Mass-41, 4/91, and D-274), AIV (H5N1, H5N2, $\mathrm{H} 5 \mathrm{~N} 3, \mathrm{H} 9, \mathrm{H} 7)$ and Adenov $\left.\left(\mathrm{EDS}_{76}\right)\right\}$. Studying the vaccination protocols in flocks under investigation and their seroconversion had led us to speculate and conclude viral affections in the different localities of Sharkia governorate. Speculating viral affections was a very hard task because the Egyptian market is jammed with a great variety of protective vaccines this was conflicting during result interpretation. Positive immune titers for AIV-H7 in sharkia governorate was detected at June $/ 2014$ in (El-salhia, 10 ${ }^{\text {th }}$ of Ramadan, El-ibrahemia and Abo-hammed) in commercial layer flocks only. The seropositive samples that exceeded the cutoff values were 63 out of 1317 (4.8\%). AIV-H9 high seropositive immune titers was constantly found in examined samples although their protective vaccines were neglected. AIV $-\mathrm{H}_{5} \mathrm{~N}_{3}$ seropositive results was recorded in a totally non vaccinated flocks against $\mathrm{H}_{5}$ which reflect virus circulation in the poultry premises. Seropositive titer for IBV-D 274 and EDS $_{76}$ was recorded in a totally non vaccinated flocks against such antigens., which refer to their role in the total simultaneous incidence of disease and consequent mortality. From another point of view it should be noted that. Vaccinating chicken flocks following a ready made manuscript of the producing companies without prior evaluation of the maternally derived antibodies (MDA) or evaluating the immune titers before taking the decision of vaccination or even considering the disease situation in the area is possible cause for vaccine failure. Sentinel birds inclusion in poultry patches should be taken seriously to give a mirror for the circulating viral agents in the poultry premises. It worth to mention that a parallel bacteriological work was running during investigation of the causes of increased mortality or dropped egg production. This work revealed the isolation of a resistant bacteria of the (Kebseilla spp).

Key words: $N D V, A I V, I B V, E D S_{76}$ Epidemiological.

\section{INTRODUCTION}

Eid (1994) stated that avian viruses causes severe economic losses in poultry beside other identified causative agents (Bacterial, mycotic, intoxication, etc.) and that the outcome of infection is influenced by many factors associated with the host organisms and environment Bradbury (1984), Dhillon and Kibenge (1987), and Gelb (1989).
Morrow (2008) stated that demonstration of antibody simply shows the antigen that a bird has been in contact with at some time in the past., but this does not prove that a clinical disease syndrome is caused by the organism associated with the particular antigen., because vaccinated flocks will have antibody from vaccination., and because natural infection could have occurred earlier. For this Paired serum samples (taken at the time of clinical disease and then in convalescence) will provide a convincing 
evidence of seroconversion and association of an agent to the clinical signs seen. As for broilers testing the seroconversion is difficult because of their short life, and for possessing maternally derived antibodies which may be from vaccination of parent stock rather than wild strain infection. For this reason sentinel birds should be grown on to allow clearer seroconversion demonstration.

Comin et al. (2013) stated that the serological diagnosis of avian influenza (AI) can be performed using different methods, yet the haemagglutination inhibition (HI) test is considered the 'gold standard' for AI antibody subtyping.

This study aimed to highlight the potential existence avian of influenza infections circulating among chicken flocks in Sharkia beside NDV, IBV and $\mathrm{EDS}_{76}$

\section{MATERIALS}

\section{Chicken flocks and Serum samples}

1317 Serum samples were collected from different localities in Sharkia Governorate from 36 poultry flocks during the period of January 2014 to November 2014., they represent (6) broiler, (2) breeder, and (28) layer chicken flocks. These flocks were suffering increased mortality, drop in egg production or assessing immune titers post vaccination. The obtained sera were stored at $-20^{\circ} \mathrm{C}$ in HI plates until used. Table (1) shows the vaccination history, source and number of the collected samples.

\section{Washed CRBCS}

Chicken RBCS were obtained from 28 day old specific antibody negative chickens (SAN).

\section{Saline}

Sodium chloride $0.9 \%$ (ADWIC) ${ }^{\circledR}$.

\section{Viral antigens}

\section{IB viral antigen}

IB viral antigens for HI test were obtained from GD Holland,

- (mass- 41- VLDA 035) lot 12639-020412 exp. 4/2022,

- (793 B Designated 4/91 - VLDIA 186) lot 13695-280613 exp.6/2023,
- (D-274 VLDIA 032) lot 12645-020812 exp. 82022 ,

\section{ND viral antigen}

Allantoic fluid from chicken embryos inoculated with Lasota NDV\{Intervet, batch-12636jj01, Exp.1-2015 \} was used as HA antigen for HI-test.

\section{$\mathrm{EDS}_{76}$ viral antigen}

Adeno 127 Designated $\mathrm{EDS}_{76}$ viral antigens for $\mathrm{HI}$ test were obtained from GD Holland. (VLDIA 038) lot 13773-020813 exp. 8-2022.

\section{AIV viral antigens}

- H5N1 (Kindly obtained from Dr. Souzan Tolba NDV department El-Abassia)

- H5N2 (lot 101111A pro. 10/11/2011 exp. 10/11/2015). Kindly obtained from Profarm for vaccine distribution.

- H5N3 (VLDIA 240 GD Holand) lot 7605010607 exp. 11-2023.

- H9 (VLDIA 113 GD Holand) lot 14672-080414 exp. 4-2024.

- H7 (VLDIA 98 GD Holand) lot 10604-260110 exp. 1-2020.

\section{Negative serum}

Sera from one day old SPF chicks were used.

\section{METHODS}

\section{HI for IBV, NDV, EDS 76}

HI tests, for the fore mentioned antigens were performed as described by Villegas (2006).

\section{HI for AIV.}

OIE Manual of Diagnostic Tests and Vaccines for Terrestrial Animals 2010.

\section{Bacteriological Examination:}

Bacteriological samples were examined according to Osbaldiston (1973).

\section{Statistical analysis.}

Data were statistically analyzed as described by Snedecor and Cochran (1967) using SPSS.16 computer program, value was used to determine significance. 
Fig. 1: location of Sharkia governorate in Egypt

\begin{tabular}{|c|c|c|c|c|}
\hline NO & $\begin{array}{l}\text { Localities of } \\
\text { Sharkia }\end{array}$ & Designation & $\begin{array}{c}\text { Samples } \\
\text { Per Locality }\end{array}$ & $\begin{array}{c}\text { Flock Sample } \\
\text { Distribution }\end{array}$ \\
\hline 1 & 10th of Ramadan & 0 & 5 & \multirow{7}{*}{$\begin{array}{l}28 \text { Layer Flock } \\
6 \text { Broiler Flock } \\
2 \text { Broiler Breeder Flock }\end{array}$} \\
\hline 2 & Abo Hammad & $n$ & 13 & \\
\hline 3 & Bilbees & $\Delta$ & 2 & \\
\hline 4 & Zagazig & $\boldsymbol{t}$ & 2 & \\
\hline 5 & El-Salhia & 0 & 12 & \\
\hline 6 & El-Ibrahemia & $\mathbf{3}$ & 1 & \\
\hline 7 & Kafer Sakker & $\Delta$ & 1 & \\
\hline
\end{tabular}

Fig. 2: Some Localities of Sharkia

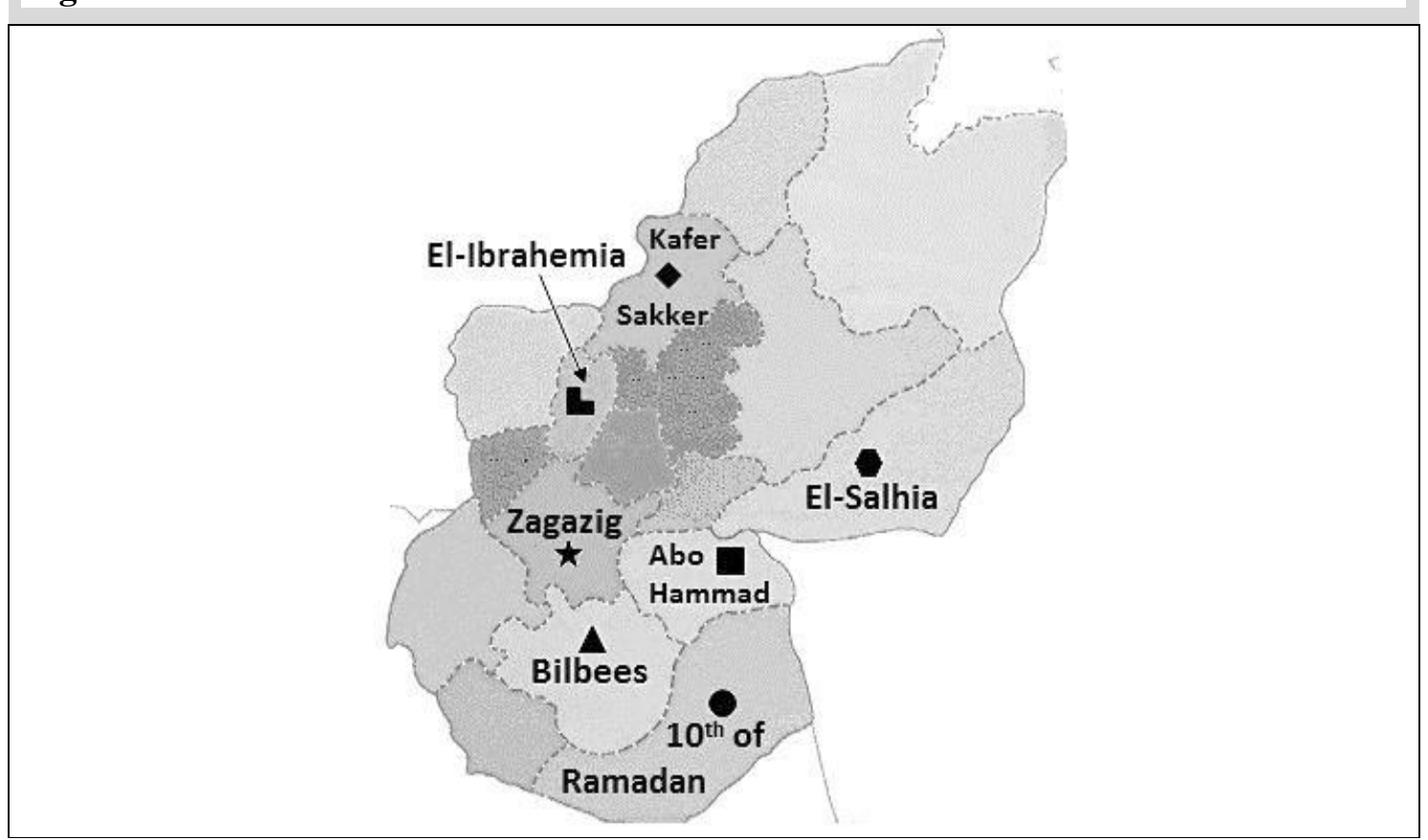


$\underline{\text { Assiut Vet. Med. J. Vol. } 61 \text { No. } 145 \text { April } 2015}$

Table 1: History of investigated samples.

\begin{tabular}{|c|c|c|c|c|c|c|c|c|c|}
\hline \multirow{2}{*}{ 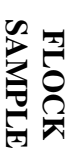 } & \multirow[b]{2}{*}{ Date } & \multirow[b]{2}{*}{ : } & \multirow[b]{2}{*}{ Z } & \multirow[b]{2}{*}{ 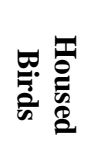 } & \multirow[b]{2}{*}{$\begin{array}{l}\mathbb{0} \\
0 \\
0 \\
0\end{array}$} & \multirow{2}{*}{ 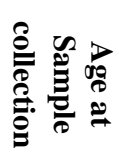 } & \multirow[b]{2}{*}{ Complain } & \multicolumn{2}{|r|}{ Vaccination history } \\
\hline & & & & & & & & 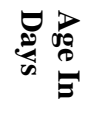 & vaccines \\
\hline \multirow[t]{10}{*}{1} & $12-1-2014$ & $\diamond$ & 23 & 6000 & $\mathbf{L}$. & $28 W$ & $\sqrt{ }$ Egg Prod. & 5D & Killed NDV, Clone - IBV $\left(\mathrm{H}_{120}\right)$ \\
\hline & & & & & & & & $13 \mathrm{D}$ & $\mathrm{H}_{5} \mathrm{~N}_{1}$ \\
\hline & & & & & & & & $16 \mathrm{D}$ & Clone - IBV $\left(\mathrm{H}_{120}\right)$ \\
\hline & & & & & & & & $28 \mathrm{D}$ & IBV $\left(\mathrm{H}_{120}\right)$ - Lasota \\
\hline & & & & & & & & $48 \mathrm{D}$ & Pox - AIV \\
\hline & & & & & & & & $65 \mathrm{D}$ & $\operatorname{IBV}\left(\mathrm{H}_{120}\right)$ - Lasota \\
\hline & & & & & & & & $80 \mathrm{D}$ & $\operatorname{IBV}\left(\mathrm{H}_{120}\right)$ - Lasota \\
\hline & & & & & & & & $100 \mathrm{D}$ & $\mathrm{H}_{5}-\mathrm{H}_{9}$ \\
\hline & & & & & & & & $105 \mathrm{D}$ & $\operatorname{IBV}\left(\mathrm{H}_{120}\right)$ - Lasota \\
\hline & & & & & & & & $125 \mathrm{D}$ & Killed (NDV + IBV + $\left.\mathbf{E D S}_{76}\right)$ \\
\hline \multirow[t]{5}{*}{2} & 19-1-2014 & $\star$ & 10 & 5000 & B. & $3 \mathbf{W}$ & 仓 Mort. & $1 \mathrm{D}$ & $H_{1}-\operatorname{IBV}\left(\mathrm{H}_{120}\right)$ \\
\hline & & & & & & & + Colisepticaemia & $9 \mathrm{D}$ & IBV ${ }_{\text {MA-5 }}$, Clone \\
\hline & & & & & & & & $10 \mathrm{D}$ & killed (NDV -AIV-H $)_{5}$ \\
\hline & & & & & & & & $14 \mathrm{D}$ & IBV $_{4 / 91}$ \\
\hline & & & & & & & & $19 \mathrm{D}$ & $\mathrm{NDV}_{6 / 10}$ \\
\hline \multirow[t]{10}{*}{3} & $10-3-2014$ & - & 75 & 75000 & L. & $13 W$ & n Prod. & $1 \mathrm{D}$ & IB $\left(\mathbf{H}_{120}\right)$ \\
\hline & & & & & & & & $6 \mathrm{D}$ & $\mathrm{HB}_{1}$ \\
\hline & & & & & & & & 8 D & $\operatorname{IBV}\left(\mathrm{H}_{120}\right)$ - Lasota \\
\hline & & & & & & & & $15 \mathrm{D}$ & IBV $_{4 / 91}$ \\
\hline & & & & & & & & $22 \mathrm{D}$ & Lasota \\
\hline & & & & & & & & $40 \mathrm{D}$ & IBV MA-5 \\
\hline & & & & & & & & $45 \mathrm{D}$ & Lasota \\
\hline & & & & & & & & $65 \mathrm{D}$ & Lasota \\
\hline & & & & & & & & $80 \mathrm{D}$ & IBV $_{4 / 91}$ \\
\hline & & & & & & & & $85 \mathrm{D}$ & Lasota \\
\hline \multirow[t]{3}{*}{4} & 28-3-2014 & $\star$ & 40 & 13500 & B. & $4 W$ & 仓 Mort. & $7 \mathrm{D}$ & $\mathrm{HB}_{1}$ \\
\hline & & & & & & & & $18 \mathrm{D}$ & H5N1 \\
\hline & & & & & & & & $20 \mathrm{D}$ & Clone \\
\hline \multirow[t]{13}{*}{5} & $31-3-2014$ & - & 75 & 75000 & L. & $61 \mathrm{~W}$ & $\sqrt{n}$ Prod. & $1 \mathrm{D}$ & IB $\left(\mathbf{H}_{120}\right)$ \\
\hline & & & & & & & & $6 \mathrm{D}$ & $\mathrm{HB}_{1}$ \\
\hline & & & & & & & & $8 \mathrm{D}$ & $\operatorname{IBV}\left(\mathrm{H}_{120}\right)$ - Lasota \\
\hline & & & & & & & & $15 \mathrm{D}$ & IBV $_{4 / 91}$ \\
\hline & & & & & & & & 22 D & Lasota \\
\hline & & & & & & & & $40 \mathrm{D}$ & IBV $V_{\text {MA-5 }}$ \\
\hline & & & & & & & & $45 \mathrm{D}$ & Lasota \\
\hline & & & & & & & & $65 \mathrm{D}$ & Lasota \\
\hline & & & & & & & & 80 D & IBV $_{4 / 91}$ \\
\hline & & & & & & & & $85 \mathrm{D}$ & Lasota \\
\hline & & & & & & & & $102 \mathrm{D}$ & $\operatorname{IBV}\left(\mathrm{H}_{120}\right)$ - Lasota \\
\hline & & & & & & & & $103 \mathrm{D}$ & Killed (NDV + IBV + EDS $\left._{76}\right)$ \\
\hline & & & & & & & & $110 \mathrm{D}$ & H5 \\
\hline 6 & $12-4-2014$ & - & 75 & 75000 & L. & $20 \mathrm{~W}$ & $\begin{array}{l}\sqrt{ } \text { Prod. } \\
\text { \&Egg deformity }\end{array}$ & G S-9 & \\
\hline \multirow[t]{4}{*}{ 7) } & $26-4-2014$ & $\boldsymbol{\Delta}$ & 35 & 5000 & L. & $26 \mathrm{~W}$ & 仓 Mort. & $1 \mathrm{D}$ & IB $\left(\mathrm{H}_{120}\right)$ \\
\hline & & & & & & & & $6 \mathrm{D}$ & $\mathrm{HB}_{1}$ \\
\hline & & & & & & & & $8 \mathrm{D}$ & IBV $\left(\mathrm{H}_{120}\right)$ - Lasota \\
\hline & & & & & & & & $15 \mathrm{D}$ & $\mathrm{IBV}_{4 / 91}$ \\
\hline
\end{tabular}


Assiut Vet. Med. J. Vol. 61 No. 145 April 2015

\begin{tabular}{|c|c|c|c|c|c|c|c|c|}
\hline \multirow{11}{*}{ 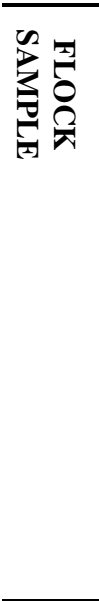 } & \multirow{11}{*}{ Date } & \multirow{11}{*}{ 完 } & \multirow{11}{*}{ 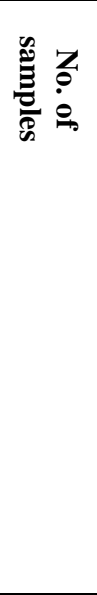 } & \multirow{11}{*}{ 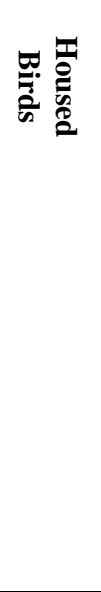 } & \multirow{11}{*}{$\begin{array}{l}\mathbb{\sigma} \\
\mathbb{0} \\
0\end{array}$} & \multirow{11}{*}{ 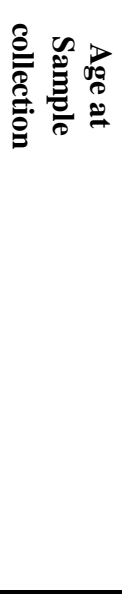 } & \multirow{11}{*}{ Complain } & Vaccination history \\
\hline & & & & & & & & 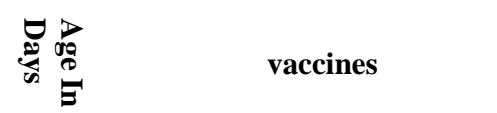 \\
\hline & & & & & & & & 22 D Lasota \\
\hline & & & & & & & & 40 D IBV MA-5 \\
\hline & & & & & & & & 45 D $\quad$ Lasota \\
\hline & & & & & & & & 65 D $\quad$ Lasota \\
\hline & & & & & & & & $80 \mathrm{D} \quad$ IBV $_{4 / 91}$ \\
\hline & & & & & & & & 85 D Lasota \\
\hline & & & & & & & & 102 D IBV $\left(H_{120}\right)$ - Lasota \\
\hline & & & & & & & & 103 D Killed (NDV + IBV + EDS $\left.{ }_{76}\right)$ \\
\hline & & & & & & & & $110 \mathrm{D} \mathrm{H}_{5}$ \\
\hline 8 & $30-4-2014$ & $\theta$ & 75 & 75000 & L. & $64 \mathrm{~W}$ & $\sqrt{ }$ Prod. & G S-9 \\
\hline \multirow[t]{6}{*}{9} & $1-5-2014$ & 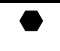 & 15 & 25000 & B. & $5 \mathrm{~W}$ & & $5 \mathrm{D} \quad \mathrm{HB}_{1}$ \\
\hline & & & & & & & & $\begin{array}{ll}6 \mathrm{D} & \mathrm{H}_{9}-\mathrm{ND} \\
\end{array}$ \\
\hline & & & & & & & & $9 \mathrm{D} \quad \mathrm{H}_{5} \mathrm{~N}_{1}$ \\
\hline & & & & & & & & 14 D IB Primer $\left(\mathrm{H}_{120}+\mathrm{D}-274\right)$ \\
\hline & & & & & & & & 17 D NDV $_{\text {Avi new }}$ \\
\hline & & & & & & & & $27 \mathrm{D} \quad \mathrm{HB}_{1}$ \\
\hline 10 & $16-5-2014$ & $\mathbf{\square}$ & 15 & 10000 & L. & $15 \mathrm{~W}$ & 仓 Mort.』 Prod. & Unknown \\
\hline \multirow[t]{2}{*}{11} & 18-5-2014 & $\overline{-1}$ & 14 & 5000 & B. & $4 \mathrm{~W}$ & 仓 Mort. & $7 \mathrm{D} \quad \mathrm{HB}_{1}-\mathrm{IB}\left(\mathrm{H}_{120}\right)$ \\
\hline & & & & & & & & 24 D $\quad$ Clone - IBV $\left(\mathrm{H}_{120}\right)$ \\
\hline \multirow[t]{2}{*}{12} & $20-5-2014$ & $\mathbf{\square}$ & 25 & 10000 & L. & $3 W$ & $\sqrt{ }$ Prod. & $7 \mathrm{D} \quad \mathrm{HB}_{1}-\mathrm{IB}\left(\mathrm{H}_{120}\right)$ \\
\hline & & & & & & & & 13 D IBV(H120) - Lasota \\
\hline \multirow[t]{3}{*}{13} & $21-5-2014$ & $\mathbf{\Delta}$ & 6 & 11000 & B. & $3 W$ & $\sqrt{n}$ Prod. & 1B Primer $\left(\mathrm{H}_{120}+\mathrm{D}-{ }_{274}\right)$ \\
\hline & & & & & & & & $\begin{array}{ll}7 \mathrm{D} & \mathrm{HB}_{1}-\mathrm{IB}\left(\mathrm{H}_{120}\right) \\
\end{array}$ \\
\hline & & & & & & & & 9D $\quad$ Clone \\
\hline 14 & $1-6-2014$ & 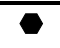 & 75 & 75000 & L. & $35 \mathrm{~W}$ & $\sqrt{n}$ Prod. & G S-9 \\
\hline 15 & $3-6-2014$ & $\mathbf{\square}$ & 14 & 4000 & B. & $4 \mathrm{~W}$ & $\sqrt{n \text { Prod. }}$ & Unkown \\
\hline \multirow[t]{13}{*}{16} & $5-6-2014$ & $\bullet$ & 35 & 58000 & L. & $20 \mathrm{~W}$ & $\sqrt{n \text { Prod. }}$ & $\begin{array}{ll}1 \mathrm{D} & \text { IB }\left(\mathrm{H}_{120}\right)\end{array}$ \\
\hline & & & & & & & & $\begin{array}{ll}6 \mathrm{D} & \mathrm{HB}_{1} \\
\end{array}$ \\
\hline & & & & & & & & $8 \mathrm{D} \quad \operatorname{IBV}\left(\mathrm{H}_{120}\right)$ - Lasota \\
\hline & & & & & & & & $15 \mathrm{D} \quad$ IBV $_{4 / 91}$ \\
\hline & & & & & & & & 22 D $\quad$ Lasota \\
\hline & & & & & & & & 40 D IBV MA-5 $_{4}$ \\
\hline & & & & & & & & 45 D $\quad$ Lasota \\
\hline & & & & & & & & 65 D $\quad$ Lasota \\
\hline & & & & & & & & $80 \mathrm{D} \quad$ IBV $_{4 / 91}$ \\
\hline & & & & & & & & 85 D $\quad$ Lasota \\
\hline & & & & & & & & 102 D IBV $\left(\mathrm{H}_{120}\right)$ - Lasota \\
\hline & & & & & & & & 103 D Killed (NDV + IBV + EDS 76$)$ \\
\hline & & & & & & & & $110 \mathrm{D} \mathrm{H}_{5}$ \\
\hline 17 & $22-6-2014$ & $\bullet$ & 15 & 11000 & L. & $22 \mathrm{~W}$ & n Prod. & Unknown \\
\hline 18 & $15-6-2014$ & $\bullet$ & 50 & 5800 & L. & $22 \mathrm{~W}$ & $\sqrt{n}$ Prod. & Unknown \\
\hline 19 & 16-6-2014 & 0 & 50 & 75000 & L. & $21 \mathrm{~W}$ & $\sqrt{n}$ Prod. & G S-9 \\
\hline 20 & 23-6-2014 & 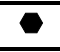 & 75 & 75000 & L. & $51 \mathrm{~W}$ & $\sqrt{n}$ Prod. & G S-9 \\
\hline 21 & $23-7-2014$ & 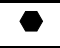 & 75 & 75000 & L. & $26 \mathrm{~W}$ & I Prod. & G S-9 \\
\hline 22 & $27-8-2014$ & 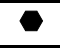 & 75 & 75000 & L. & $31 \mathrm{~W}$ & $\sqrt{1}$ Prod. & G S-9 \\
\hline \multirow[t]{3}{*}{23} & 31-8-2014 & $\mathbf{\square}$ & 20 & 20000 & L. & $10 \mathrm{~W}$ & $\sqrt{n}$ Prod. & $7 \mathrm{D} \quad \mathrm{HB}_{1}-\mathrm{IB}\left(\mathrm{H}_{120}\right)$ \\
\hline & & & & & & & & 10 D KILLED (NDV + IBDV ) \\
\hline & & & & & & & & 21 D Clone - IBV $\left(\mathrm{H}_{120}\right)$ \\
\hline
\end{tabular}




\begin{tabular}{|c|c|c|c|c|c|c|c|}
\hline \multirow{2}{*}{ 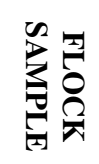 } & \multirow[b]{2}{*}{ Date } & \multirow[b]{2}{*}{ 占 } & \multirow[b]{2}{*}{ 总 } & \multirow[b]{2}{*}{ 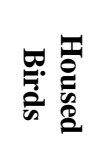 } & \multirow{2}{*}{ 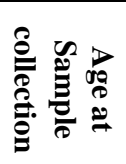 } & \multirow[b]{2}{*}{ Complain } & Vaccination history \\
\hline & & & & & & & 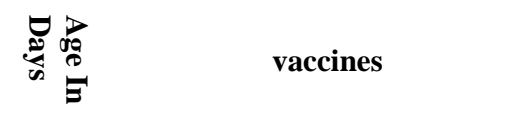 \\
\hline 24 & 9-9-2014 & $\bullet$ & 40 & 40000 L. & $35 \mathrm{~W}$ & $\sqrt{3}$ Prod. & Unknown \\
\hline 25 & $17-9-2014$ & $\bullet$ & 20 & 58000 L. & 33W & 仓 Mort. , ㄱ. Prod. & Unknown \\
\hline \multirow[t]{8}{*}{26} & 21-9-2014 & 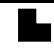 & 15 & 2000 L. & 30W & $*$ & $7 \mathrm{D} \quad \mathrm{HB}_{1}-\mathrm{IB}\left(\mathrm{H}_{120}\right)$ \\
\hline & & & & & & & 19 D Clone \\
\hline & & & & & & & 40 D Clone- IB $\left(\mathrm{H}_{120}\right)$ \\
\hline & & & & & & & $60 \mathrm{D} \quad$ Clone \\
\hline & & & & & & & 90 D Clone - IB $\left(\mathrm{H}_{120}\right)$ \\
\hline & & & & & & & 100 D Killed (NDV + IBV + EDS 76$)$ \\
\hline & & & & & & & 110 D Clone \\
\hline & & & & & & & 130 D Lasota \\
\hline \multirow[t]{9}{*}{27} & $10-9-2014$ & घ & 20 & 10000 L. & $42 W$ & $\sqrt{1}$ Prod. & $7 \mathrm{D} \quad \mathrm{HB}_{1}-\mathrm{IB}\left(\mathrm{H}_{120}\right)$ \\
\hline & & & & & & & 10 D KILLED ( NDV + IBDV) \\
\hline & & & & & & & 21 D $\quad$ Clone - IBV $\left(\mathrm{H}_{120}\right)$ \\
\hline & & & & & & & 65 D $\quad$ Lasota \\
\hline & & & & & & & 80 D $\quad$ IBV $_{4 / 91}$ \\
\hline & & & & & & & 85 D Lasota \\
\hline & & & & & & & 102 D IBV $\left(H_{120}\right)$ - Lasota \\
\hline & & & & & & & 103 D Killed (NDV + IBV + EDS 76$)$ \\
\hline & & & & & & & $110 \mathrm{D} \mathrm{H}_{5}$ \\
\hline \multirow[t]{3}{*}{28} & 27-9-2014 & च & 15 & 10000 L. & $15 \mathrm{~W}$ & $\sqrt{l}$ Prod. & $7 \mathrm{D} \quad \mathrm{HB}_{1}-\mathrm{IBV}\left(\mathrm{H}_{120}\right)$ \\
\hline & & & & & & & 10 D KILLED ( NDV + IBDV) \\
\hline & & & & & & & 21 D Clone + IBV $\left(\mathrm{H}_{120}\right)$ \\
\hline 29 & $30-9-2014$ & $\mathbf{\square}$ & 45 & 13000 B.B. & $32 \mathrm{~W}$ & $*$ & Unknown \\
\hline 30 & 6-10-2014 & 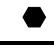 & 75 & 75000 L. & $21 \mathrm{~W}$ & $\sqrt{n}$ Prod. & G S-9 \\
\hline \multirow[t]{3}{*}{31} & $12-10-2014$ & च & 20 & 18000 B.B. & $32 \mathrm{~W}$ & $*$ & $20 \mathrm{~W}$ KILLED (ND, IB, VA, IBDV) \\
\hline & & & & & & & 23 W Lasota \\
\hline & & & & & & & $25 \mathrm{~W} \quad \mathrm{H}_{5} \mathrm{~N}_{1}$ \\
\hline 32 & 15-10-2014 & 口 & 20 & 4000 L. & $11 \mathrm{~W}$ & $*$ & Unknown \\
\hline 33 & $12-10-2014$ & 口 & 20 & 3000 L. & $15 \mathrm{~W}$ & $\sqrt{1}$ Prod. & Unknown \\
\hline 34 & 21-10-2014 & a & 20 & 5000 L. & $20 \mathrm{~W}$ & $\sqrt{n}$ Prod. & Unknown \\
\hline 35 & 29-10-2014 & $\mathbf{\square}$ & 20 & 5000 L. & $25 \mathrm{~W}$ & $\sqrt{n}$ Prod. & Unknown \\
\hline 36 & 3-11-2014 & $\mathbf{\square}$ & 20 & 3000 L. & $31 \mathrm{~W}$ & $\begin{array}{l}\sqrt{3} \text { Prod \& } \\
\text { Mort. }\end{array}$ & Unknown \\
\hline \multicolumn{4}{|c|}{$\mathrm{L}=$ Layer } & B. $=$ Broiler & & & B.B.= Broiler Breeder \\
\hline \multicolumn{8}{|c|}{ G S-9 An integrated egg producing company applying a fixed vaccination protocol } \\
\hline \multicolumn{8}{|c|}{ Unknown $=$ unknown vaccination history } \\
\hline \multicolumn{8}{|c|}{$*=$ Evaluation of vaccination titers. } \\
\hline
\end{tabular}

\section{RESULT}

Results of the present work are illustrated in tables (2-4). 
Assiut Vet. Med. J. Vol. 61 No. 145 April 2015

Table 2: Results of Serological investigations (After Cut-off values)

\begin{tabular}{|c|c|c|c|c|c|c|c|c|c|c|c|}
\hline \multirow{3}{*}{ 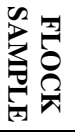 } & & \multicolumn{10}{|c|}{ Laboratory Works } \\
\hline & & \multirow{2}{*}{ NDV } & \multicolumn{5}{|c|}{ AIV } & \multicolumn{3}{|c|}{ IBV } & \multirow{2}{*}{$\mathbf{E D S}_{76}$} \\
\hline & & & H5N1 & H5N2 & H5N3 & H9 & H7N1 & Mass-41 & $4 / 91$ & D-274 & \\
\hline \multirow{3}{*}{1} & $*$ & $\begin{array}{c}2.61 \pm \\
0.41\end{array}$ & $\begin{array}{c}2.43 \pm \\
0.56\end{array}$ & $\begin{array}{c}9.78 \pm \\
0.11\end{array}$ & \multirow{3}{*}{$\mathbf{0}$} & $\begin{array}{c}9.16 \pm \\
0.24\end{array}$ & \multirow{3}{*}{$\mathbf{0}$} & $\begin{array}{c}4.78 \pm \\
0.11\end{array}$ & $\begin{array}{c}2.35 \pm \\
0.54\end{array}$ & $\begin{array}{c}7.19 \pm \\
0.19\end{array}$ & \multirow{3}{*}{$\mathbf{0}$} \\
\hline & $* *$ & 1.95 & 2.69 & 0.52 & & 1.21 & & 0.52 & 2.59 & 0.75 & \\
\hline & $* * *$ & 3.79 & 7.26 & 0.27 & & 1.47 & & 0.27 & 6.69 & 0.56 & \\
\hline \multirow{3}{*}{2} & $*$ & $\begin{array}{l}7.6 \pm \\
0.52\end{array}$ & $\begin{array}{c}6.62 \pm \\
0.18\end{array}$ & $\begin{array}{c}4 \pm \\
0.93\end{array}$ & \multirow{3}{*}{$\mathbf{0}$} & $\begin{array}{c}0.8 \pm \\
0.53\end{array}$ & \multirow{3}{*}{$\mathbf{0}$} & $\begin{array}{c}1.9 \pm \\
0.52\end{array}$ & $\begin{array}{c}6.63 \pm \\
0.18\end{array}$ & $\begin{array}{c}8.75 \pm \\
0.59\end{array}$ & $\begin{array}{c}6.75 \pm \\
0.16\end{array}$ \\
\hline & $* *$ & 1.65 & 0.65 & 2.94 & & 1.69 & & 1.66 & 0.52 & 1.67 & 0.46 \\
\hline & $* * *$ & 2.71 & 0.42 & 8.67 & & 2.84 & & 2.77 & 0.27 & 2.79 & 0.21 \\
\hline \multirow{3}{*}{3} & $*$ & $\begin{array}{c}7.84 \\
\pm 0.23 \\
\end{array}$ & $\begin{array}{c}8.04 \pm \\
0.14\end{array}$ & $\begin{array}{c}6.78 \pm \\
0.21\end{array}$ & $\begin{array}{c}9.98 \pm \\
0.02\end{array}$ & $\begin{array}{c}5.45 \pm \\
0.4\end{array}$ & \multirow{3}{*}{$\mathbf{0}$} & $\begin{array}{c}8.68 \pm \\
0.17\end{array}$ & $\begin{array}{c}2.5 \pm \\
0.34\end{array}$ & $\begin{array}{c}5.52 \pm \\
0.08\end{array}$ & $\begin{array}{c}9.13 \pm \\
0.21\end{array}$ \\
\hline & $* *$ & 1.95 & 1.21 & 1.81 & 0.13 & 3.45 & & 1.5 & 2.52 & 0.57 & 1.58 \\
\hline & $* * *$ & 3.81 & 1.47 & 3.27 & 0.02 & 11.93 & & 2.25 & 6.33 & 0.33 & 2.51 \\
\hline \multirow{3}{*}{4} & $*$ & $\begin{array}{c}3.25 \\
\pm 1.11\end{array}$ & $\begin{array}{c}2.75 \pm \\
1.6\end{array}$ & $\begin{array}{c}8.38 \pm \\
0.46\end{array}$ & \multirow{3}{*}{$\mathbf{0}$} & $\begin{array}{c}5.25 \pm \\
0.25\end{array}$ & \multirow{3}{*}{$\mathbf{0}$} & $\begin{array}{c}3.75 \pm \\
1.25\end{array}$ & \multirow{3}{*}{$\mathbf{0}$} & \multirow{3}{*}{$\mathbf{0}$} & \multirow{3}{*}{$\mathbf{0}$} \\
\hline & $* *$ & 2.22 & 3.2 & 1.3 & & 0.5 & & 2.5 & & & \\
\hline & $* * *$ & 4.92 & 10.25 & 1.7 & & 0.25 & & 6.25 & & & \\
\hline \multirow{3}{*}{5} & $*$ & $\begin{array}{c}8.74 \\
\pm 0.14\end{array}$ & $\begin{array}{c}6.39 \pm \\
0.2\end{array}$ & $\begin{array}{c}5.78 \pm \\
0.29\end{array}$ & \multirow{3}{*}{ 0 } & $\begin{array}{c}9.25 \pm \\
0.15\end{array}$ & \multirow{3}{*}{ 0 } & $\begin{array}{c}4.96 \pm \\
0.02\end{array}$ & & & \\
\hline & $* *$ & 1.19 & 1.72 & 2.46 & & 1.3 & & 0.2 & 0 & 0 & 0 \\
\hline & $* * *$ & 1.43 & 2.97 & 6.05 & & 1.68 & & 0.04 & & & \\
\hline 6 & $*$ & $\begin{array}{c}9.45 \\
\pm 0.13\end{array}$ & $6 \pm 0$ & $\begin{array}{c}7.79 \pm \\
0.25\end{array}$ & $\begin{array}{c}9.95 \pm \\
0.05\end{array}$ & $\begin{array}{c}9.18 \pm \\
0.23\end{array}$ & 0 & $\begin{array}{c}9.92 \pm \\
0.08\end{array}$ & $\begin{array}{c}1.88 \pm \\
0.41\end{array}$ & $\begin{array}{c}4.75 \pm \\
0.18\end{array}$ & $\begin{array}{c}7.98 \pm \\
0.17 \\
\end{array}$ \\
\hline & $* *$ & 1.14 & 0 & 2.16 & 0.32 & 1.47 & & 0.4 & 2.62 & 1.17 & 1.07 \\
\hline & $* * *$ & 1.31 & 0 & 4.68 & 0.1 & 2.15 & & 0.16 & 6.88 & 1.37 & 1.15 \\
\hline 7 & $*$ & $\begin{array}{c}9.69 \pm \\
0.1\end{array}$ & $\begin{array}{c}7.31 \pm \\
0.22\end{array}$ & $\begin{array}{l}4.6 \pm \\
0.22\end{array}$ & $\begin{array}{c}8.04 \pm \\
0.54\end{array}$ & $\begin{array}{c}8.83 \pm \\
0.19\end{array}$ & 0 & $\begin{array}{c}2.56 \pm \\
0.22\end{array}$ & $\begin{array}{c}2.09 \pm \\
0.34\end{array}$ & $\begin{array}{c}5.02 \pm \\
0.16\end{array}$ & $\begin{array}{c}7.09 \pm \\
0.3\end{array}$ \\
\hline & $* *$ & 0.58 & 1.33 & 0.70 & 4.01 & 1.11 & & 1.32 & 2.58 & 1.2 & 2.28 \\
\hline & $* * *$ & 0.33 & 1.76 & 0.49 & 16.07 & 1.23 & & 1.74 & 6.66 & 1.44 & 5.21 \\
\hline 8 & $*$ & $\begin{array}{c}3.53 \\
\pm 0.41\end{array}$ & $\begin{array}{c}6.4 \pm \\
0.27\end{array}$ & $\begin{array}{c}8.68 \pm \\
0.16\end{array}$ & $\begin{array}{l}9.5 \pm \\
0.35\end{array}$ & $\begin{array}{c}9.53 \pm \\
0.28\end{array}$ & 0 & $\begin{array}{c}3.79 \pm \\
0.07\end{array}$ & $\begin{array}{c}2.38 \pm \\
0.41\end{array}$ & $\begin{array}{l}5.2 \pm \\
0.18\end{array}$ & $\begin{array}{c}3.25 \pm \\
0.45\end{array}$ \\
\hline & $* *$ & 2.31 & 1.06 & 1.38 & 2.21 & 1.74 & & 0.58 & 2.59 & 1.11 & 2.85 \\
\hline & $* * *$ & 5.35 & 1.11 & 1.9 & 4.87 & 3.03 & & 0.33 & 6.7 & 1.24 & 8.14 \\
\hline & $*$ & $\begin{array}{c}4.93 \\
\pm 0.07 \\
\end{array}$ & $\begin{array}{c}6.33 \pm \\
0.25\end{array}$ & $\begin{array}{c}7.75 \pm \\
0.41\end{array}$ & $\begin{array}{c}7.7 \pm \\
0.26\end{array}$ & $10 \pm 0$ & & $\begin{array}{c}4.38 \pm \\
0.33\end{array}$ & $\begin{array}{c}1.93 \pm \\
0.65\end{array}$ & $\begin{array}{c}6.69 \pm \\
0.21\end{array}$ & \\
\hline 9 & $* *$ & 0.26 & 0.98 & 1.42 & 0.82 & 0 & 0 & 1.31 & 2.52 & 0.75 & 0 \\
\hline & $* * *$ & 0.07 & 0.95 & 2.02 & 0.68 & $\mathbf{0}$ & & 1.72 & 6.35 & 0.56 & \\
\hline & * & $\begin{array}{c}4.73 \\
\pm 0.12\end{array}$ & $\begin{array}{c}5.8 \pm \\
0.14\end{array}$ & $\begin{array}{c}3.47 \pm \\
0.7\end{array}$ & $\begin{array}{c}3.5 \pm \\
0.66\end{array}$ & $\begin{array}{c}9.67 \pm \\
0.16\end{array}$ & & $\begin{array}{c}6 \pm \\
0.16\end{array}$ & $\begin{array}{c}6.74 \pm \\
0.27\end{array}$ & $\begin{array}{c}7.88 \pm \\
0.48\end{array}$ & $\begin{array}{c}4.4 \pm \\
0.16\end{array}$ \\
\hline 10 & $* *$ & 0.46 & 0.56 & 2.72 & 2.47 & 0.62 & 0 & 0.58 & 1.01 & 1.36 & 0.52 \\
\hline & $* * *$ & 0.21 & 0.31 & 7.41 & 6.12 & 0.38 & & 0.33 & 1.02 & 1.84 & 0.27 \\
\hline & $*$ & $\begin{array}{c}6.75 \\
\pm 0.63\end{array}$ & $10 \pm 0$ & $\begin{array}{l}7.5 \pm \\
0.46\end{array}$ & & $1 \pm 1$ & & & & $\begin{array}{c}3.43 \pm \\
0.14\end{array}$ & $5 \pm 0$ \\
\hline 11 & $* *$ & 1.26 & 0 & 1.31 & 0 & 2 & U & 0 & 0 & 0.51 & 0 \\
\hline & $* * *$ & 1.58 & 0 & 1.71 & & 4 & & & & 0.26 & 0 \\
\hline & * & $\begin{array}{c}6.88 \pm \\
0.7\end{array}$ & $10 \pm 0$ & & & $\begin{array}{c}0.32 \pm \\
0.22\end{array}$ & & $\begin{array}{c}1.32 \pm \\
0.3\end{array}$ & $\begin{array}{c}2.71 \pm \\
0.54\end{array}$ & & \\
\hline 12 & $* *$ & 3.48 & 0 & $\mathbf{0}$ & $\mathbf{0}$ & 1.11 & $\mathbf{0}$ & 1.52 & 2.63 & 0 & 0 \\
\hline & $* * *$ & 12.11 & $\mathbf{0}$ & & & 1.23 & & 2.31 & 6.91 & & \\
\hline
\end{tabular}


$\underline{\text { Assiut Vet. Med. J. Vol. } 61 \text { No. } 145 \text { April } 2015}$

\begin{tabular}{|c|c|c|c|c|c|c|c|c|c|c|c|}
\hline \multirow{3}{*}{ 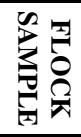 } & & \multicolumn{10}{|c|}{ Laboratory Works } \\
\hline & & \multirow{2}{*}{ NDV } & \multicolumn{5}{|c|}{ AIV } & \multicolumn{3}{|c|}{ IBV } & \multirow{2}{*}{$\mathbf{E D S}_{76}$} \\
\hline & & & H5N1 & H5N2 & H5N3 & H9 & H7N1 & Mass-41 & $4 / 91$ & D-274 & \\
\hline \multirow{3}{*}{13} & $*$ & \multirow{3}{*}{$\mathbf{0}$} & \multirow{3}{*}{$\mathbf{0}$} & \multirow{3}{*}{$\mathbf{0}$} & $\begin{array}{c}9.88 \pm \\
0.12\end{array}$ & $\begin{array}{c}1.67 \pm \\
1.05\end{array}$ & \multirow{3}{*}{$\mathbf{0}$} & $5 \pm 0$ & \multirow{3}{*}{$\mathbf{0}$} & \multirow{3}{*}{$\mathbf{0}$} & $7 \pm 0.41$ \\
\hline & $* *$ & & & & 0.35 & 2.58 & & 0 & & & 0.82 \\
\hline & $* * *$ & & & & 0.13 & 6.67 & & $\mathbf{0}$ & & & 0.67 \\
\hline \multirow{3}{*}{14} & $*$ & $\begin{array}{c}9.65 \pm \\
0.1\end{array}$ & $\begin{array}{c}6.25 \pm \\
0.16\end{array}$ & \multirow{3}{*}{$\mathbf{0}$} & $\begin{array}{l}8.7 \pm \\
0.38\end{array}$ & $\begin{array}{c}9.66 \pm \\
0.15\end{array}$ & $1 \pm 0.25$ & \multirow{3}{*}{ 0 } & $\begin{array}{c}2.91 \pm \\
0.35\end{array}$ & $\begin{array}{c}4.64 \pm \\
0.15\end{array}$ & $\begin{array}{c}6.02 \\
\pm 0.41 \\
\end{array}$ \\
\hline & $* *$ & 0.89 & 0.46 & & 2.85 & 1.15 & 1.86 & & 2.62 & 1.15 & 3.1 \\
\hline & $* * *$ & 0.8 & 0.21 & & 8.11 & 1.32 & 3.45 & & 6.85 & 1.32 & 9.58 \\
\hline \multirow{3}{*}{15} & $*$ & $10 \pm 0$ & $5 \pm 0$ & \multirow{3}{*}{ 0 } & $\begin{array}{c}4.36 \pm \\
0.34 \\
\end{array}$ & $\begin{array}{c}0.83 \pm \\
0.57 \\
\end{array}$ & \multirow{3}{*}{$\mathbf{0}$} & $5 \pm 0$ & $\begin{array}{c}1.29 \pm \\
0.57 \\
\end{array}$ & $\begin{array}{c}3.18 \pm \\
0.18 \\
\end{array}$ & \multirow{3}{*}{$\mathbf{0}$} \\
\hline & $* *$ & $\overline{0}$ & $\mathbf{0}$ & & 1.28 & 1.99 & & $\overline{0}$ & 2.13 & 0.73 & \\
\hline & $* * *$ & $\mathbf{0}$ & $\mathbf{0}$ & & 1.63 & 3.97 & & $\mathbf{0}$ & 4.53 & 0.53 & \\
\hline \multirow{3}{*}{16} & $*$ & $\begin{array}{c}6.24 \\
\pm 0.29 \\
\end{array}$ & $\begin{array}{c}0.38 \pm \\
0.18 \\
\end{array}$ & \multirow{3}{*}{$\mathbf{0}$} & \multirow{3}{*}{$\mathbf{0}$} & $\begin{array}{c}4.95 \pm \\
0.65 \\
\end{array}$ & & $\begin{array}{c}4.97 \pm \\
0.03 \\
\end{array}$ & $\begin{array}{c}2.71 \pm \\
0.46 \\
\end{array}$ & & \\
\hline & $* *$ & 1.88 & 1.19 & & & 4.24 & $\mathbf{0}$ & 0.17 & 2.74 & $\mathbf{0}$ & $\mathbf{0}$ \\
\hline & $* * *$ & 3.55 & 1.41 & & & 17.95 & & 0.03 & 7.5 & & \\
\hline 17 & $*$ & $\begin{array}{c}8.13 \pm \\
0.6\end{array}$ & $7 \pm 0.29$ & $\begin{array}{c}6.53 \pm \\
0.73\end{array}$ & $\begin{array}{c}9.75 \pm \\
0.16\end{array}$ & $10 \pm 0$ & $\begin{array}{c}3.88 \pm \\
0.89\end{array}$ & $\begin{array}{c}7 \pm \\
0.36\end{array}$ & $\begin{array}{c}2.13 \pm \\
1.07\end{array}$ & $\begin{array}{c}5 \pm \\
0.27\end{array}$ & $\begin{array}{c}4.13 \pm \\
1.24\end{array}$ \\
\hline & $* *$ & 2.33 & 1.13 & 2.83 & 0.46 & 0 & 2.53 & 1.15 & 3.04 & 0.76 & 3.52 \\
\hline & $* * *$ & 5.41 & 1.29 & 7.98 & 0.21 & 0 & 6.41 & 1.33 & 9.27 & 0.57 & 12.41 \\
\hline 18 & $*$ & $\begin{array}{c}8.98 \\
\pm 0.34\end{array}$ & $10 \pm 0$ & & $\begin{array}{c}9.1 \pm \\
0.24\end{array}$ & $\begin{array}{c}9.64 \pm \\
0.15\end{array}$ & $\begin{array}{l}0.1 \pm \\
0.01\end{array}$ & $\begin{array}{c}5.85 \pm \\
0.19\end{array}$ & $\begin{array}{c}1.68 \pm \\
0.37\end{array}$ & $\begin{array}{c}4.9 \pm \\
0.15\end{array}$ & $\begin{array}{c}5.02 \pm \\
0.56\end{array}$ \\
\hline & $* *$ & 2.38 & 0 & O & 1.72 & 1.08 & 0.71 & 1.1 & 2.62 & 1.05 & 3.98 \\
\hline & $* * *$ & 5.95 & $\mathbf{0}$ & & 2.95 & 1.17 & 0.5 & 1.22 & 6.88 & 1.11 & 15.86 \\
\hline 19 & $*$ & $\begin{array}{c}9.48 \pm \\
0.1 \\
\end{array}$ & $\begin{array}{c}8.12 \pm \\
0.26 \\
\end{array}$ & & $\begin{array}{c}7.09 \pm \\
0.44 \\
\end{array}$ & $\begin{array}{c}9.67 \pm \\
0.12 \\
\end{array}$ & $\begin{array}{l}0.5 \pm \\
0.24 \\
\end{array}$ & $\begin{array}{c}4.24 \pm \\
0.1 \\
\end{array}$ & $\begin{array}{c}2.48 \pm \\
0.36 \\
\end{array}$ & $\begin{array}{c}3.89 \pm \\
0.3 \\
\end{array}$ & $\begin{array}{c}6.52 \pm \\
0.55 \\
\end{array}$ \\
\hline 18 & $* *$ & 0.68 & 1.3 & $\mathbf{0}$ & 2.97 & 0.82 & 1.5 & 0.72 & 2.45 & 2.01 & 3.76 \\
\hline & $* * *$ & 0.46 & 1.69 & & 8.84 & 0.67 & 2.26 & 0.51 & 5.99 & 4.05 & 14.12 \\
\hline 20 & $*$ & $\begin{array}{c}8.43 \\
\pm 0.21 \\
\end{array}$ & $\begin{array}{l}6.4 \pm \\
0.25\end{array}$ & & $\begin{array}{c}8.3 \pm \\
0.3\end{array}$ & $\begin{array}{c}9.27 \pm \\
0.17\end{array}$ & $\begin{array}{c}0.11 \pm \\
0.11\end{array}$ & $\begin{array}{c}7.67 \pm \\
0.22\end{array}$ & $\begin{array}{c}3.39 \pm \\
0.35\end{array}$ & $\begin{array}{c}4.98 \pm \\
0.16\end{array}$ & $\begin{array}{c}4.96 \pm \\
0.51\end{array}$ \\
\hline & $* *$ & 1.85 & 0.55 & $\mathbf{0}$ & 2.27 & 1.26 & 0.8 & 1.85 & 2.63 & 1.2 & 3.85 \\
\hline & $* * *$ & 3.44 & 0.30 & & 5.16 & 1.58 & 0.64 & 3.44 & 6.93 & 1.44 & 14.8 \\
\hline & $*$ & $\begin{array}{c}8.89 \\
\pm 0.13\end{array}$ & $\begin{array}{l}4 \pm \\
22\end{array}$ & & $\begin{array}{c}7.95 \pm \\
0.17\end{array}$ & $\begin{array}{c}9.73 \pm \\
0.07\end{array}$ & $\begin{array}{c}0.1 \pm \\
0.1\end{array}$ & $\begin{array}{c}6.5 \pm \\
0.15\end{array}$ & $\begin{array}{c}1.95 \pm \\
0.41\end{array}$ & $\begin{array}{c}5.63 \pm \\
0.09\end{array}$ & $\begin{array}{c}4.2 \pm \\
0.48\end{array}$ \\
\hline 21 & $* *$ & 1.1 & 0.58 & 0 & 1.08 & 0.62 & 0.63 & 0.52 & 2.59 & 0.59 & 3.01 \\
\hline & $* * *$ & 1.2 & 0.33 & & 1.18 & 0.39 & 0.4 & 0.27 & 6.72 & 0.34 & 9.09 \\
\hline 22 & $*$ & $\begin{array}{c}8.78 \\
\pm 0.17\end{array}$ & $\begin{array}{l}7.9 \pm \\
0.23\end{array}$ & & & $\begin{array}{c}6.76 \pm \\
0.16\end{array}$ & & $\begin{array}{c}.92 \pm \\
0.04\end{array}$ & & & \\
\hline & $* *$ & 1.22 & 0.74 & $\mathbf{0}$ & 0 & 1.41 & $\mathbf{0}$ & 0.27 & 0 & $\mathbf{0}$ & $\mathbf{0}$ \\
\hline & $* * *$ & 1.48 & 0.54 & & & 2 & & 0.08 & & & \\
\hline 23 & $*$ & $\begin{array}{l}9.1 \pm \\
0.51 \\
\end{array}$ & $\begin{array}{c}1.17 \pm \\
0.43 \\
\end{array}$ & $\begin{array}{l}7.5 \pm \\
0.46 \\
\end{array}$ & & $\begin{array}{c}9.83 \pm \\
0.1 \\
\end{array}$ & & $\begin{array}{c}8.87 \pm \\
0.32 \\
\end{array}$ & & & \\
\hline & $* *$ & 1.6 & 2.1 & 1.31 & 0 & 0.48 & 0 & 1.25 & 0 & 0 & 0 \\
\hline & $* * *$ & 2.54 & 4.41 & 1.71 & & 0.23 & & 1.55 & & & \\
\hline 24 & $*$ & $\begin{array}{c}9.53 \pm \\
0.2\end{array}$ & $\begin{array}{l}5.1 \pm \\
0.48\end{array}$ & & $\begin{array}{c}7.56 \pm \\
0.52 \\
\end{array}$ & $\begin{array}{c}9.75 \pm \\
0.15\end{array}$ & 0 & $\begin{array}{c}4.41 \pm \\
0.45\end{array}$ & $\begin{array}{c}2.63 \pm \\
0.46\end{array}$ & $\begin{array}{c}5.47 \pm \\
0.11\end{array}$ & $\begin{array}{c}5.38 \pm \\
0.62\end{array}$ \\
\hline & $* *$ & 1.24 & 3.05 & $\mathbf{0}$ & 2.96 & 0.84 & & 2.55 & 2.59 & 0.62 & 3.5 \\
\hline & $* * *$ & 1.54 & 9.32 & & 8.77 & 0.71 & & 6.51 & 6.69 & 0.39 & 12.24 \\
\hline 25 & $*$ & $\begin{array}{c}9 \pm \\
0.16\end{array}$ & $\begin{array}{c}3.4 \pm \\
0.7\end{array}$ & 0 & $\begin{array}{c}9 \pm \\
0.34\end{array}$ & $\begin{array}{c}7.05 \pm \\
0.21\end{array}$ & 0 & $\begin{array}{l}7.2 \pm \\
0.29\end{array}$ & $\begin{array}{c}2.69 \pm \\
0.71\end{array}$ & $\begin{array}{c}.06 \pm \\
0.49\end{array}$ & $\begin{array}{c}9.08 \pm \\
0.43\end{array}$ \\
\hline & $* *$ & 0.73 & 3.12 & & 1.37 & 0.94 & & 0.92 & 2.85 & 1.95 & 2.12 \\
\hline
\end{tabular}


Assiut Vet. Med. J. Vol. 61 No. 145 April 2015

\begin{tabular}{|c|c|c|c|c|c|c|c|c|c|c|c|}
\hline \multirow{4}{*}{ 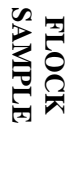 } & & \multicolumn{10}{|c|}{ Laboratory Works } \\
\hline & & \multirow{2}{*}{ NDV } & \multicolumn{5}{|c|}{ AIV } & \multicolumn{3}{|c|}{ IBV } & \multirow{2}{*}{$\mathbf{E D S}_{76}$} \\
\hline & & & H5N1 & H5N2 & H5N3 & H9 & H7N1 & Mass-41 & $4 / 91$ & D-274 & \\
\hline & $* * *$ & 0.53 & 9.73 & & 1.87 & 0.89 & & 0.84 & 8.1 & 3.8 & 4.51 \\
\hline \multirow{3}{*}{26} & $*$ & $\begin{array}{c}9.8 \pm \\
0.11 \\
\end{array}$ & \multirow{3}{*}{ 0 } & $6 \pm 0.5$ & $\begin{array}{c}7.75 \pm \\
0.37 \\
\end{array}$ & $10 \pm 0$ & \multirow[t]{3}{*}{ 0 } & $3 \pm 0$ & $\begin{array}{c}4.63 \pm \\
0.71 \\
\end{array}$ & $\begin{array}{l}5.5 \pm \\
0.27 \\
\end{array}$ & $\begin{array}{c}9.63 \pm \\
0.37 \\
\end{array}$ \\
\hline & $* *$ & 0.41 & & 1.93 & 1.04 & $\mathbf{0}$ & & $\mathbf{0}$ & 2 & 0.76 & 1.06 \\
\hline & $* * *$ & 0.17 & & 3.71 & 1.07 & $\mathbf{0}$ & & $\mathbf{0}$ & 3.98 & 0.57 & 1.13 \\
\hline \multirow{3}{*}{27} & $*$ & $10 \pm 0$ & $\begin{array}{c}3.88 \pm \\
0.41 \\
\end{array}$ & \multirow{3}{*}{$\mathbf{0}$} & $\begin{array}{c}8.19 \pm \\
0.67 \\
\end{array}$ & $10 \pm 0$ & \multirow{3}{*}{ 0 } & $\begin{array}{l}9.2 \pm \\
0.25 \\
\end{array}$ & $\begin{array}{c}1.94 \pm \\
0.66 \\
\end{array}$ & $\begin{array}{c}5.44 \pm \\
0.18 \\
\end{array}$ & $\begin{array}{c}9.94 \pm \\
0.06 \\
\end{array}$ \\
\hline & $* *$ & $\mathbf{0}$ & 2.03 & & 2.66 & $\mathbf{0}$ & & 0.79 & 2.62 & 0.73 & 0.25 \\
\hline & $* * *$ & $\mathbf{0}$ & 4.11 & & 7.1 & $\mathbf{0}$ & & 0.62 & 6.86 & 0.53 & 0.06 \\
\hline \multirow{3}{*}{28} & $*$ & $\begin{array}{c}8.93 \\
\pm 0.33 \\
\end{array}$ & $\begin{array}{l}5.8 \pm \\
0.24 \\
\end{array}$ & \multirow{3}{*}{$\mathbf{0}$} & $\begin{array}{c}9.92 \pm \\
0.08 \\
\end{array}$ & $10 \pm 0$ & $\begin{array}{c}4.54 \pm \\
0.32 \\
\end{array}$ & $\begin{array}{c}4.93 \pm \\
0.07 \\
\end{array}$ & $\begin{array}{c}1.67 \pm \\
0.54 \\
\end{array}$ & \multirow{3}{*}{ 0 } & $\begin{array}{c}8.88 \pm \\
0.32 \\
\end{array}$ \\
\hline & $* *$ & 1.28 & 0.94 & & 0.41 & $\mathbf{0}$ & 1.56 & 0.26 & 2.66 & & 1.57 \\
\hline & $* * *$ & 1.64 & 0.89 & & 0.17 & $\mathbf{0}$ & 2.43 & 0.07 & 7.1 & & 2.46 \\
\hline \multirow{3}{*}{29} & $*$ & $10 \pm 0$ & $\begin{array}{c}9.09 \pm \\
0.2\end{array}$ & \multirow{3}{*}{$\mathbf{0}$} & $10 \pm 0$ & $10 \pm 0$ & $\begin{array}{c}0.25 \pm \\
0.25\end{array}$ & $\begin{array}{c}4.64 \pm \\
0.19\end{array}$ & $\begin{array}{c}3.44 \pm \\
0.63\end{array}$ & $\begin{array}{c}4.88 \pm \\
0.16\end{array}$ & $\begin{array}{c}9 \pm \\
0.26\end{array}$ \\
\hline & $* *$ & $\mathbf{0}$ & 1.35 & & 0 & 0 & 1 & 1.26 & 2.53 & 0.62 & 1.03 \\
\hline & $* * *$ & $\mathbf{0}$ & 1.81 & & $\mathbf{0}$ & $\mathbf{0}$ & 1 & 1.6 & 6.4 & 0.38 & 1.07 \\
\hline \multirow{3}{*}{30} & $*$ & $10 \pm 0$ & $10 \pm 0$ & $10 \pm 0$ & $\begin{array}{c}9.69 \pm \\
0.22 \\
\end{array}$ & $\begin{array}{c}9.99 \pm \\
0.01 \\
\end{array}$ & $\begin{array}{c}1.75 \pm \\
0.32 \\
\end{array}$ & $\begin{array}{c}3.77 \pm \\
0.11 \\
\end{array}$ & $\begin{array}{c}3.06 \pm \\
0.38 \\
\end{array}$ & $\begin{array}{c}4.33 \pm \\
0.2 \\
\end{array}$ & $\begin{array}{c}5.13 \pm \\
0.37 \\
\end{array}$ \\
\hline & $* *$ & $\mathbf{0}$ & $\mathbf{0}$ & 0 & 1.49 & 0.12 & 2.24 & 0.94 & 2.6 & 1.39 & 2.56 \\
\hline & $* * *$ & $\mathbf{0}$ & $\mathbf{0}$ & 0 & 2.22 & 0.01 & 5 & 0.88 & 6.74 & 1.93 & 6.54 \\
\hline \multirow{3}{*}{31} & $*$ & $\begin{array}{c}8.08 \pm \\
0.4 \\
\end{array}$ & $\begin{array}{c}1.46 \pm \\
0.67 \\
\end{array}$ & \multirow{3}{*}{ 0 } & $\begin{array}{c}7.62 \pm \\
0.33 \\
\end{array}$ & $10 \pm 0$ & \multirow{3}{*}{ 0 } & $\begin{array}{c}6.88 \pm \\
0.3 \\
\end{array}$ & $\begin{array}{c}3.54 \pm \\
0.81 \\
\end{array}$ & $\begin{array}{c}4.69 \pm \\
0.24 \\
\end{array}$ & $\begin{array}{c}6.62 \pm \\
0.9 \\
\end{array}$ \\
\hline & $* *$ & 1.44 & 2.4 & & 1.19 & 0 & & 1.32 & 2.93 & 0.85 & 3.23 \\
\hline & $* * *$ & 2.08 & 5.77 & & 1.42 & $\mathbf{0}$ & & 1.74 & 8.6 & 0.73 & 10.42 \\
\hline \multirow{3}{*}{32} & $*$ & $\begin{array}{c}9.97 \\
\pm 0.03 \\
\end{array}$ & $7 \pm 0.3$ & $\begin{array}{c}3.21 \pm \\
0.59\end{array}$ & $\begin{array}{c}6.27 \pm \\
0.27 \\
\end{array}$ & $\begin{array}{c}9.73 \pm \\
0.12\end{array}$ & \multirow[t]{3}{*}{ 0 } & $\begin{array}{c}6.74 \pm \\
0.27\end{array}$ & $\begin{array}{c}1.93 \pm \\
0.65\end{array}$ & $\begin{array}{c}5.67 \pm \\
0.13\end{array}$ & $\begin{array}{c}6.8 \pm \\
0.8\end{array}$ \\
\hline & $* *$ & 0.18 & 1.11 & 2.19 & 1.03 & 0.46 & & 1.01 & 2.52 & 0.49 & 3.1 \\
\hline & $* * *$ & 0.03 & 1.23 & 4.8 & 1.07 & 0.21 & & 1.02 & 6.35 & 0.24 & 9.6 \\
\hline \multirow{3}{*}{33} & $*$ & $\begin{array}{c}3.88 \\
\pm 1.34 \\
\end{array}$ & $10 \pm 0$ & & $\begin{array}{l}5.5 \pm \\
0.38 \\
\end{array}$ & $\begin{array}{c}8.13 \pm \\
0.12 \\
\end{array}$ & & & $\begin{array}{l}1.5 \pm \\
0.73 \\
\end{array}$ & $\begin{array}{c}3.88 \pm \\
0.23 \\
\end{array}$ & \begin{tabular}{|l}
$7.5 \pm$ \\
0.46 \\
\end{tabular} \\
\hline & $* *$ & 3.8 & $\mathbf{0}$ & 0 & 1.07 & 0.35 & 0 & 0 & 2.07 & 0.64 & 1.31 \\
\hline & $* * *$ & 14.41 & $\mathbf{0}$ & & 1.14 & 0.13 & & & 4.29 & 0.41 & 1.71 \\
\hline 34 & $*$ & $\begin{array}{c}5.38 \\
\pm 0.15 \\
\end{array}$ & $\begin{array}{c}\mathbf{3 . 3 3} \pm \\
\mathbf{0 . 8 7} \\
\end{array}$ & & $\begin{array}{l}8.8 \pm \\
0.57 \\
\end{array}$ & $\begin{array}{l}9.3 \pm \\
0.34 \\
\end{array}$ & $\begin{array}{l}3.2 \pm \\
0.71 \\
\end{array}$ & $\begin{array}{c}8.85 \pm \\
0.3 \\
\end{array}$ & $\begin{array}{l}2.1 \pm \\
0.71 \\
\end{array}$ & $\begin{array}{c}4.2 \pm \\
0.59 \\
\end{array}$ & $\begin{array}{l}6.1 \pm \\
1.08 \\
\end{array}$ \\
\hline & $* *$ & 0.83 & 2.6 & 0 & 1.81 & 1.06 & 2.25 & 1.07 & 2.23 & 1.87 & 3.41 \\
\hline & $* * *$ & 0.69 & 6.75 & & 3.29 & 1.12 & 5.07 & 1.14 & 4.99 & 3.51 & 11.66 \\
\hline 35 & $*$ & $\begin{array}{c}9.46 \\
\pm 0.28 \\
\end{array}$ & $\begin{array}{c}.75 \pm \\
0.66 \\
\end{array}$ & & $10 \pm 0$ & $\begin{array}{c}9.65 \pm \\
0.21 \\
\end{array}$ & $\begin{array}{c}1.35 \pm \\
0.48\end{array}$ & $\begin{array}{c}3 \pm \\
0.52 \\
\end{array}$ & $\begin{array}{c}1.25 \pm \\
0.51 \\
\end{array}$ & $\begin{array}{c}5.4 \pm \\
0.18 \\
\end{array}$ & \begin{tabular}{|l|}
$5.3 \pm$ \\
0.51 \\
\end{tabular} \\
\hline & $* *$ & 1.38 & 2.65 & 0 & 0 & 0.93 & 2.13 & 1.81 & 2.27 & 0.82 & 2.27 \\
\hline & $* * *$ & 1.91 & 7 & & $\mathbf{0}$ & 0.87 & 4.56 & 3.27 & 5.14 & 0.67 & 5.17 \\
\hline 36 & $*$ & $\begin{array}{c}6.92 \\
\pm 0.22\end{array}$ & $\begin{array}{c}7.38 \pm \\
0.18\end{array}$ & & $10 \pm 0$ & $\begin{array}{c}9.83 \pm \\
0.17\end{array}$ & $\begin{array}{l}1.5 \pm \\
0.96\end{array}$ & $\begin{array}{c}9.1 \pm \\
0.28\end{array}$ & $1 \pm 1$ & $\begin{array}{c}5.83 \pm \\
0.17\end{array}$ & $4 \pm 0$ \\
\hline & $* *$ & 1.08 & 0.52 & 0 & $\mathbf{0}$ & 0.41 & 2.35 & 0.88 & 2.45 & 0.41 & $\mathbf{0}$ \\
\hline & $* * *$ & 1.16 & 0.27 & & $\mathbf{0}$ & 0.17 & 5.5 & 0.77 & 6 & 0.17 & $\mathbf{0}$ \\
\hline & & verage & & $* *$ Stand & Deviation & & $* * *$ & ariance & & $\mathbf{0}=\mathrm{Nc}$ & Done \\
\hline
\end{tabular}

Table 3: Simultaneous viral affections in the examined samples 
$\underline{\text { Assiut Vet. Med. J. Vol. } 61 \text { No. } 145 \text { April } 2015}$

\begin{tabular}{|c|c|c|c|c|c|c|c|}
\hline No. & $\begin{array}{l}\text { Predicted viral } \\
\text { affections }\end{array}$ & No. & Predicted viral affections & No. & $\begin{array}{l}\text { Predicted viral } \\
\text { affections }\end{array}$ & No. & Predicted viral affections \\
\hline 1 & IBV $_{\text {D-274 }}$ & 10 & $\mathrm{H9}+\mathrm{IBV}_{\mathrm{D}-274}$ & 19 & $\mathrm{NDV}+\mathrm{H} 7+\mathrm{H9}+\mathrm{IBV}_{\mathrm{D}-274}$ & 28 & $\mathbf{H} 7+\mathrm{H} 9 \mathrm{EDS}_{76,+} \mathrm{H}_{5} \mathbf{N}_{3}$ \\
\hline 2 & $\mathrm{H9}+\mathrm{EDS}_{76}+\mathrm{IBV}_{\mathrm{D}-274}$ & 11 & $\mathrm{H9}+\mathrm{EDS}_{76}+\mathrm{IBV}_{\mathrm{D}-274}+\mathrm{IBV}_{\mathrm{D}-274}$ & 20 & H7+H9 & 29 & $\mathrm{NDV}+\mathrm{H} 7+\mathrm{H} 9 \mathrm{EDS}_{76,+} \mathrm{H}_{5} \mathrm{~N}_{3}$ \\
\hline 3 & $\mathrm{H9}+\mathrm{IBV}_{\mathrm{D}-274}+\mathrm{H}_{5} \mathrm{~N}_{3}$ & 12 & H9 & 21 & $\mathrm{H} 7+\mathrm{H} 9+\mathrm{IBV}_{\mathrm{D}-274}$ & 30 & $\mathrm{NDV}+\mathrm{H} 7+\mathrm{H} 9+\mathrm{IBV}_{\mathrm{D}-274}+\mathrm{H}_{5} \mathrm{~N}_{3}$ \\
\hline 4 & H9 & 13 & $\mathbf{H 9}+\mathrm{EDS}_{76+} \mathrm{H}_{5} \mathbf{N}_{3}$ & 22 & H9 & 31 & $\mathbf{H 9}, \mathbf{E D S}_{76}$ \\
\hline 5 & $\mathrm{H} 9+\mathrm{H}_{5} \mathrm{~N}_{3}$ & 14 & $\mathrm{NDV}+\mathrm{H} 7+\mathrm{H} 9+\mathrm{IBV}_{\mathrm{D}-274}$ & 23 & $\mathrm{NDV}+\mathrm{H} 9$ & 32 & $\mathrm{NDV}+\mathrm{H9}$ \\
\hline 6 & $\mathrm{NDV}+\mathrm{H9}$ & 15 & H9 & 24 & $\mathrm{NDV}+\mathrm{H} 9+\mathrm{IBV}_{\mathrm{D}-274}$ & 33 & H9 \\
\hline 7 & $\mathrm{NDV}+\mathrm{H9}$ & 16 & H9 & 25 & $\mathrm{H9}, \mathrm{EDS}_{76}$ & 34 & H7+H9 \\
\hline 8 & $\mathrm{H9}+\mathrm{IBV}_{\mathrm{D}-274}+\mathrm{H}_{5} \mathrm{~N}_{3}$ & 17 & $\mathrm{H} 7+\mathrm{H} 9+\mathrm{IBV}_{\mathrm{D}-274}+\mathrm{H}_{5} \mathrm{~N}_{3}$ & 26 & H9, $\mathrm{EDS}_{76}+\mathrm{IBV}_{\mathrm{D}-274}$ & 35 & $\mathrm{NDV}+\mathrm{H} 7+\mathrm{H} 9+\mathrm{H}_{5} \mathrm{~N}_{3}$ \\
\hline 9 & H9 & 18 & $\mathrm{H} 7+\mathrm{H} 9+\mathrm{IBV}_{\mathrm{D}-274}+\mathrm{H}_{5} \mathrm{~N}_{3}$ & 27 & $\mathrm{NDV}+\mathrm{H9}, \mathrm{EDS}_{76}+\mathrm{IBV}_{\mathrm{D}-274}$ & 36 & $\mathrm{H} 7+\mathrm{H} 9+\mathrm{H}_{5} \mathrm{~N}_{3}$ \\
\hline
\end{tabular}

Table 4: Viral Concurrency in relation to El- Sharkia localities

\begin{tabular}{|c|c|c|c|}
\hline NO. & Localities of sharkia & Designation & Predicted viral affections \\
\hline 1 & 10th of Ramadan & 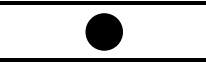 & $\mathrm{H9}, \mathrm{H} 7, \mathrm{EDS}_{76}, \mathrm{IBV}_{\mathrm{D}-274}+\mathrm{H}_{5} \mathrm{~N}_{3}$ \\
\hline 2 & Abo Hammad & $\square_{2}$ & H9,H7,NDV, $\mathrm{EDS}_{76}, \mathrm{IBV}_{\mathrm{D}-274}$ \\
\hline 3 & Bilbees & $\Delta$ & 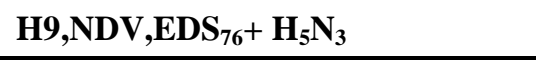 \\
\hline 4 & Zagazig & $\boldsymbol{t}$ & $\mathrm{H9}, \mathrm{EDS}_{76}, \mathrm{IBV}_{\mathrm{D}-274}$ \\
\hline 5 & El-Salhia & 4 & $\mathrm{H9}, \mathrm{H} 7, \mathrm{NDV}, \mathrm{EDS}_{76}, \mathrm{IBV}_{\mathrm{D}-274}+\mathrm{H}_{5} \mathrm{~N}_{3}$ \\
\hline 6 & El-Ibrahemia & ד & H9, EDS $_{76}$, IBV V$_{\mathrm{D}-274}$ \\
\hline
\end{tabular}

\section{DISCUSSION}

Morrow (2008) stated that., demonstration of antibody through seroservillance simply shows what a bird has been in contact with at some time in the past., but this does not prove that a clinical syndrome is caused by a certain antigen., because vaccinated flocks will have antibody from vaccination., and natural infection could have occurred earlier and was not associated with this clinical syndrome. For these reasons a paired serum samples, taken at the time of clinical disease and in convalescence, provides the most convincing evidence of seroconversion and association of an agent to the clinical signs seen. He also mentioned that, testing the seroconversion in broilers is difficult because of their short life span, and for possessing maternally derived antibodies. For this reason sentinel birds should be grown on without neglicance., to allow clearer seroconversion demonstration.

During interpretation of serologic data It is usually impossible to differentiate between antibodies that are produced by vaccination or those resulting from field exposure to a given infectious agent. The only difference that may be observed is that the antibody titer following a field challenge may be higher than that observed following vaccination. A valid interpretation of serologic results requires a complete knowledge of the flock's vaccination history and disease situation. In the present study a detailed vaccination history is illustrated in table (1). Morrow (2008) stated that., the number of serum samples needed depends on the reason for the testing and test characteristics i.e. for diagnosis., 10-60 serum samples per group should be taken., smaller numbers can be taken from sentinel birds during paired sampling for demonstration of the seroconversion., more samples are required to show an overall decrease in the number of seronegative birds., and for confirmation of freedom from infection a maximum of 60 samples per group is needed to give $95 \%$ confidence that infection of $5 \%$ of the animals would be detected. In this case the observation of one positive result defines the group as infected. This sort of testing can be a part of an eradication programme. He also mentioned that for post vaccination response evaluation a 20-30 sera per group are enough.

Newcastle disease virus (NDV) is one of the highly contagious diseases contained in the list $\mathrm{A}$ of the Office International des Epizooties (OIE). The disease is caused by the avian paramyxovirus serotype-1 (APMV-1). It affects a variety of avian species but causes most severe disease and economic losses in domestic poultry Kaleta (1992). Infection with different strains of NDV may result in a broad variation in severity and spread of the disease, even in a single host species.

Beard and Hanson (1981). Grouped NDV strains into five pathotypes based on the induced clinical signs in infected chickens (i) viscerotropic velogenic (associated with high mortality and intestinal lesions). (ii) Neurotropic velogenic (associated with high mortality following central-nervous signs). (iii) 
mesogenic (associated with low mortality, respiratory and nervous signs). (iv) lentogenic (associated with mild or clinical inapparent respiratory infections). (v) Asymptomatic enteritic (associated with inapparent intestinal infections).

ND is almost indistinguishable from HPAI Alexander (1997), Easterday et al., 1997). The possibility of misdiagnosing HPAI as ND couldn't be ruled out in the field. When NDV infects chickens, antibody titers rise within 6 - 10 days and subsequently diminish slowly to zero Alexander (1997). Velogenic strains usually elicit a higher titer than mesogenic and lentogenic strains Alexander (1997), Alexander et al. (2004). In non-vaccinated chickens, HI titers specific to NDV can be taken as a sign of previous infection, and titers as high as $10 \log _{2}$ suggests field exposure to pathogenic NDV Chrysostome et al. (1995), Alexander et al. (2004).

From another point of view Allan et al. (1978) were using ND- HI titer (using 4 HA units) as a predictive tools for the expected mortality and drop in egg production upon challenge as follow.

- When All individual HI values was $2^{2}$ or less ( $100 \%$ mortality on challenge is expected).

- When all individual HI values was $2^{2}$ to $2^{5} \log$ mean $2^{3.75}$ STDV 0.4 (10\% mortality on challenge is expected).

- When all individual $\mathrm{HI}$ values was $2^{4}$ to $2^{6} \log$ mean $2^{5.2}$ STDV $0.35(0 \%$ mortality on challenge is expected).

- When all individual HI values was $2^{6}$ to $2^{8} \log$ mean $2^{6.5}$ STDV 1.2 (A serious drop in egg production, with no deaths could be expected and convalescent $\mathrm{HI}$ titer $2^{14}$ or greater could be reached).

- when All individual HI values was $2^{9}$ to $2^{11} \log$ mean $2^{10.5}$ STDV 1.4 (No drop in egg production, no deaths, and a convalescent titers $2^{11}$ to $2^{12}$ could be obtained),

- And finally if the range was $2^{11}$ to $2^{13} \log$ mean $2^{11.2}$ STDV 1.3 (A flock that will remain free from any risk of NDV).

In the present study., the ND-HI titers were ranging from (0 up to 10) $\log 2$ (table-2). The titer of sample $(6,7,14,19,23,24,27,29,30,32$,and 35$)$ were ranging from (9.1 up to 10$) \log _{2}$ (table -2$)$., this could justify a NDV affection especially if we consider that the vaccines applied for these flocks is not sufficient to elect such titer.

AIV-(H5) Comin et al. (2013) found that HI test has a near perfect accuracy that might be considered as a gold standard test. The test enables processing large amounts of samples in a short time, if the reference viral antigen is close enough to the virus isolate to be tested when panels of different antigens are used. In the present study, we had used H5 with three distinct neuraminidases i.e. (H5N1, H5N2, and H5N3) these represent the AIV commercially available viral antigens in the Egyptian market.

Timm Harder (2012): In a personal communication mentioned that, the neuraminidases has a significant role in $\mathrm{HI}$ as well. He explained., that neuraminidases are enzyme that binds to its substrate, the sialic glycans that are also used as the cellular receptor for influenza A virus HA proteins. He also gave a further interpretation as follow. (Given the situation of a duck holding that had experienced an $\mathrm{H}_{11} \mathrm{~N}_{1}$ infection. Serum taken from these birds three weeks later will react in $\mathrm{HI}$ with the $\mathrm{H}_{5} \mathrm{~N}_{1}$ and the $\mathrm{H}_{7} \mathrm{~N}_{1}$ antigens but none of them will react with the $\mathrm{H}_{5} \mathrm{~N}_{3}$ or the $\mathrm{H}_{7} \mathrm{~N}_{7}$ ones. Thus such sera are considered $\mathrm{H}_{5} / \mathrm{H}_{7}$ negative despite their reactivity with one $\mathrm{H}_{5} / \mathrm{H}_{7}$ antigen!). In addition, he mentioned that $\mathrm{HI}$ assay are difficult to perform and more difficult to interpret! This is because HA and NA are always linked to diagnostic antigens (inactivated viruses). On the other hand, HI assays are highly specific. Thus, even antibodies raised against one of the Egyptian $\mathrm{H}_{5} \mathrm{~N}_{1}$ cluster 2.2.1.1 viruses will probably not react with (European) $\mathrm{H}_{5} \mathrm{~N}_{1}$ antigen due to the large antigenic distance between these HA antigens. In the present study, samples were collected from vaccinated bird. Vaccines in Egypt represent these viral categories $\left(\mathrm{H}_{5} \mathrm{~N}_{1}, \mathrm{H}_{5} \mathrm{~N}_{2}, \mathrm{H}_{5} \mathrm{~N}_{3}\right)$ in a reassortant form., so it became very difficult for us to evaluate to obtained results and because poultry holders had stopped the inclusion of sentinel birds in the breaded poultry batches., we thought that the high $\mathrm{HI}$ titer for the $\mathrm{H}_{5} \mathrm{~N}_{3}$ antigen observed in samples $(3,6,8,13,17$, $18,28,29,30,35$ and 35 ) was relevant to the vaccinal strain used as mentioned by Timm Harder (2012)., but on second thought we had found that flock sample (3,6,8 and 30) were applying vaccination against AIV using the $\mathrm{H}_{5} \mathrm{~N}_{1}$ reasssortants and flock sample $(13,28)$ were totally not vaccinated against AIV and that flock samples $(17,18,29,35$ and 36) had anonymous history., this may reflect $\mathrm{H}_{5} \mathrm{~N}_{3}$ circulation in the fore mentioned flock samples.

AIV-(H7) Abdel whab et al. (2013) mentioned that Avian influenza viruses of $\mathrm{H}_{5}$ and $\mathrm{H}_{7}$ subtypes exhibit two different pathotypes in poultry: infection with low pathogenic (LP) strains results in minimal, if any, health disturbances, whereas highly pathogenic (HP) strains cause severe morbidity and mortality. LPAIV of $\mathrm{H}_{5}$ and $\mathrm{H}_{7}$ subtypes can spontaneously mutate into HPAIV. Ten outbreaks caused by HPAIV were preceded by circulation of a predecessor or LPAIV in poultry. Three of them were caused by $\mathrm{H}_{5} \mathrm{~N}_{2}$ subtype and seven involved $\mathrm{H}_{7}$ in combination with $\mathrm{N}_{1}, \mathrm{~N}_{3}$, or $\mathrm{N}_{7}$. Abdelwhab et al. (2014) mentioned that., $\mathrm{H}_{7}$ subtype HA gene has been found in combination with all nine NA subtype genes. Most exhibit low pathogenicity and only rarely high pathogenicity in poultry (and humans). During the past few years 
infections of poultry and humans with $\mathrm{H}_{7}$ subtypes have increased markedly. In the present study., fortunately $\mathrm{H}_{7}$ vaccines is not yet allowed to Egypt., this had facilitated our mission in interpreting the obtained results, which aim to ascertain its existance in Sharkia., although the GMT of the HI against $\mathrm{H}_{7} \mathrm{~N}_{1}$ was ranging from $(.1 \pm .01-4.54 \pm .32)$ (table -2). It worth to mention., that examined samples revealed positive seroreactive values that exceeded the cut off values which could be interpreted surely as infection in samples $(14,17,18,19,20,21,28,29,30,34,35$ and 36) had a positive seroreactive that exceeded the cutoff values OIE (2010). This gives a sure incidence of AIV-H7 in different localities of sharkia governorate but this incidence was milder than the previously recorded incidence by Afifi et al. (2013). The incidence of the disease in sharkia based on a seropositive reactors could be speculated to start in (June, July, Sept., Oct., and November 2014) in the locations from which samples were collected such as (El-Salhia, 10th of Ramadan, El-Ibrahemia and Abo Hammad) (table 2 figure 1), it also worth to mention that the positive seroreactives were totally from commercial layers except for flock (sample 29) which were collected from a broiler breeder flock. It should be also noted that the total seropositive samples exceeding the cutoff values were $(63 / 1317$ with a percent of $4.78 \%$ ).

Abdel whab et al. (2014) mentioned that wild birds are the natural reservoir of the $\mathrm{H}_{7}$ virus. Geographically, the most prevalent subtype is $\mathrm{H}_{7} \mathrm{~N}_{7}$, which is endemic in wild birds in Europe and was frequently reported in domestic poultry, whereas subtype $\mathrm{H}_{7} \mathrm{~N}_{3}$ is mostly isolated from the Americas. In humans, mild to fatal infections were caused by subtypes $\mathrm{H}_{7} \mathrm{~N}_{2}, \mathrm{H}_{7} \mathrm{~N}_{3}, \mathrm{H}_{7} \mathrm{~N}_{7}$ and $\mathrm{H}_{7} \mathrm{~N}_{9}$.

While, infections of humans have been associated mostly with exposure to domestic poultry, infections of poultry have been linked to wild birds or live bird markets. Fred Leung (2012) in a personal communication commented on the role of wild migratory bird in AIV disease transfer as follow (although the virus movements were recorded in the known migratory bird routes but in the opposite direction for the migratory bird flow route). Generally, depopulation of infected poultry was the main control tool. In contrast to recent cases caused by subtype $\mathrm{H}_{7} \mathrm{~N}_{9}$, human infections were usually selflimiting and rarely required antiviral medication. Close genetic and antigenic relatedness of $\mathrm{H}_{7}$ viruses of different origins may be helpful in development of universal vaccines and diagnostics for both animals and humans. Due to the wide spread of $\mathrm{H}_{7}$ viruses and their zoonotic importance more research is required for better understanding of the epidemiology, pathobiology and virulence determinants of these viruses.
AIV-(H9) Positive seroreactive samples were recorded in the submitted samples from different localities of Sharkia governorate starting from (Jan 2014). The GMT of HI titers was ranging from (.32 up to 10). Even. In (sample15) $2 / 24$ sample exceeded the cutoff values. Observing these results and considering that only flock sample (1 and 9) had received vaccines against $\mathrm{H}_{9} \mathrm{~N}_{2}$ lead us to conclude., a wide distribution of $\mathrm{H}_{9}$ in Sharkia governorate, it also should be pointed for the fact that the distance between vaccination in flocks (1) and sample collection exceeded 100 day this could justify infection and with flock (9) we can observe that birds were simultaneously vaccinated with (AIV $\mathrm{H}_{9}+\mathrm{NDV}$ ) and that the serologic response for NDV is very low compared to the serologic response for AIV-H9 which suggests superinfection with $\mathrm{H} 9$. The total seropositive sample for $\mathrm{H} 9$ were (736 out of 1317 examined) i.e.(55.8\%).

Afifi et al. (2013). Examined the potential existence of $\mathrm{H}_{7}$ and $\mathrm{H}_{9}$ AIV circulating among chicken flocks in Egypt. Serum samples were collected from chicken flocks that experienced respiratory distresses and/or variable mortality rates. $\mathrm{H}_{7}$ and $\mathrm{H}_{9}$ virus infections were screened by HI assay. Concerning Sharkia governorate. A 133 serum samples were collected from one broiler, one breeder and two layer flocks. 52 out of 133 examined sera, seropositve i.e. (39\%) were recorded for $\mathrm{H}_{7}$.As for $\mathrm{H}_{9}$ A 113 out of 133 seropositive sample were recorded i.e. (84 \%). Prevalence of both $\mathrm{H}_{7}$ and $\mathrm{H}_{9}$ antibodies were higher in layer followed by breeder then broiler flocks. Afifi et al. (2013) concluded that special consideration should be paid to control influenza viruses in Egypt, as pandemic influenza strains may develop unnoticed given the presence of subclinical infections, and the possibility of re-assortment with the prevailing endemic $\mathrm{H}_{5} \mathrm{~N}_{1}$ virus strains exciting in Egypt.

Afifi et al. (2013) mentioned that., they were able to isolate $\mathrm{H}_{9} \mathrm{~N}_{2}$ from broiler flock from Alexandria Governorate in the northern part of Egypt (unpublished data) however, only a broiler flock from Beni-Suef was found seropositive to $\mathrm{H}_{9}$. Interestingly, another recent report for the isolation of $\mathrm{H}_{9} \mathrm{~N}_{2}$ from quail in Egypt was also reported El-Zoghby et al. (2012). Meanwhile, the isolation of AIV subtype $\mathrm{H}_{7}$ from Egypt was only recorded from migratory birds Aly et al. (2010) and Soliman et al. (2012) as follow., on $2004\left(\mathrm{H}_{7} \mathrm{~N}_{1}\right)$. On 2004, 2005, and $2006\left(\mathrm{H}_{7} \mathrm{~N}_{7}\right)$. On $2006\left(\mathrm{H}_{7} \mathrm{~N}_{9}\right)$, and on $2007\left(\mathrm{H}_{7} \mathrm{~N}_{3}\right)$. Interestingly, $\mathrm{H}_{7}$ serological results on backyard from 11 villages in the nearby areas were negative Aly et al. (2008). To the best of our knowledge, no recent report revealed isolation of $\mathrm{H}_{7}$ from commercial or backyard poultry populations. But historically, LPAIV A/turkey/ Egypt/88 $\left(\mathrm{H}_{7} \mathrm{~N}_{1}\right)$ was isolated Khafagy et al. (1992); however, they reported the absence of $\mathrm{H}_{7}$ seropositive sera when testing 6124 chicken and 92 turkey sera 
Khafagy et al. (1995). However, the presences of antibodies against AIV H7 were reported Afifi et al. (1999). Egypt is located in the pathway of migratory birds and represents a hinge zone of wild bird migration, where the East Africa-West Asia and Black Sea-Mediterranean's flyways overlap and large diversity of species migrating to and from South Africa, Europe, and Central Asia were detected in Egypt Soliman et al. (2012). It was recorded that the migratory birds plays a role in the introduction but not the spread of AIV to other wild and domestic species that are present in their migratory pathways Feare (2010), Soliman et al. (2012). Increased numbers of seropositive were observed in farms located within the migratory route of wild birds AlNatour and Abo-Shehada (2005).

Avian adenovirus (AAV) associated with clinical disease was isolated from an outbreak of respiratory disease in quail Olson (1950). Since that time, AAVs were seen in all types and breeds of chickens and from a variety of other avian species. AAV were frequently isolated from the respiratory and/or intestinal tracts of the apparently healthy chickens, and their role in the etiology of clinical disease was regarded as insignificant Yates et al. (1976) and Winterfield (1984). Sometimes., AAVs were associated with a variety of specific disease such as (quail bronchitis, inclusion body hepatitis (IBH), egg drop syndrome (EDS), turkey hemorrhagic enteritis (THE), marble spleen disease and respiratory manifestation) Du Bose et al. (1958), Du Boes and Grumbles (1959), Ismail (1966), Ahmed and El-Sisi (1969), Fadly and WinterfieId (1973), Rosenberger et al. (1974), Hoffmann et al. (1975), McFerran (1981), McFerran (1989), McFerran (1991) and McFerran and Stuart (1990). Papanikoloau et al. (1985) and McFerran and Stuart 1990). Specific pathogenicity for poultry and vertical transmission had led AAVs to receive more attention McFerran and Stuart (1990). AAV should not be neglected as complicating factors in the course of some poultry diseases e.g Mycoplasmosis and IBV Monereal and Ahmed (1963), Monereal (1966), Monereal (1968), Awad et al. (1973), Dhillon and Kibenge (1987)., and in birds vaccinated with spray IBV at day-old or immune-suppressed by IBDV Mousa et al. (1984), McFerran and Stuart (1990).

Egg drop syndrome $1976\left(\mathrm{EDS}_{76}\right)$ is an economically important viral disease characterized by a severe drop in egg production as well as the production of shell less, thin-shelled, discolored or misshapen eggs Van Ecz (1982). Although, the occurrence of $\mathrm{EDS}_{76}$ in layers has been reported worldwide but there is limited information on its occurrence in broiler chickens Meulemans et al. (1979).

Hemagglutinating AAV is represented by $\mathrm{EDS}_{76}$. There are three evident patterns of $\mathrm{EDS}_{76}$. Classical $\mathrm{EDS}_{76}$ following the introduction of virus into primary breeding stock, probably through a vaccine grown in duck cells. Spread was through the embryo and the resulting reactivation of virus at peak of egg production gave an apparent breed and age susceptibility. The second pattern is the endemic form where lateral spread between flocks via contaminated eggs occurs at any age of laying hens. The third (sporadic) form is again seen in any age or breed of birds resulting from infection from ducks and geese or any infected wild bird McFerran and Stuart (1990).

To our knowledge, reports on seroprevalence of $\mathrm{EDS}_{76}$ in chickens in commercial farms in Sharkia are scanty. Although killed vaccines against $\mathrm{EDS}_{76}$ such as $\left(\mathrm{IBV}+\mathrm{NDV}+\mathrm{EDS}_{76}\right)$ or $\left(\mathrm{IBV}+\mathrm{NDV}+\mathrm{EDS}_{76}+\mathrm{AIV}\right)$ are used in commercial layer flocks. In the present study three broiler flocks (sample 2), (sample 11) and (sample 13) were serologically positive and had HI titre of $(6.75 \pm 0.16,5$ and 7$)$ respectively after neglecting the cutoff values., these flocks were located in (zagazig, Belbees and El-salhia). On the other hand., layer flocks in (sample 6), (sample 7) and sample 8) had a seropositive HI titer at age of $20 \mathrm{wk}$, $26 \mathrm{wk}$, and $26 \mathrm{wk}$ of age that was $\{7.98 \pm 0.17,7.09 \pm$ 0.3 and $3.25 \pm 0.45\}$ respectively., these flocks had received the triple killed vaccine at age around 11 WK of age during the rearing period in integrated plants but the layer flocks in small holders such as flock (sample 25), (sample 26), (sample 27), (sample 28), (sample 29) and (sample 31) had HI titer ranging from $(6.62 \pm 0.9$ up to $9.94 \pm .06)$ which could be referred to as an infection. These flock were located in (Belbees, 10 $0^{\text {th }}$ of Ramadan, El-Ibrahemia and Abo-hammad).

Singh et al. (1995) screened 22 broiler flocks using 347 serum samples examined with the (HI) test., they found that 114 sample i.e. $(32.9 \%)$ were positive for antibodies to $\left(\operatorname{EDS}_{76}\right)$, the $\mathrm{HI}$ titres of these serum samples ranged from 2 to $9 \log 2$ and the overall geometric mean titre was $3.9 \log 2$. All of the examined flocks in the present study were positive for antibodies suggesting widespread EDS $_{76}^{\prime}$ infection in this region of Egypt. The presence of high HI antibody titres suggests that infection was acquired at an early age and this could not be due to maternal antibodies as the present study was limited to birds 5 to 10 weeks old and maternally derived antibodies are known to persist only up to 3 weeks of age McFerran (1981), McFerran and Stuart (1990). Vertical transmission is common in $\mathrm{EDS}_{76}^{\prime}$ virus infection but congenitally infected birds may not become serologically positive until 25-28 weeks of age VanEcz (1982). Because it has been observed that congenital infection remains dormant until sexual maturity or in response to stress of production Nawathe and Abegunde (1980). Contrary to this the present observation of $\mathrm{HI}$ antibody in broiler birds provides evidence for horizontal spread of the virus. This is supported by other workers who have 
confirmed the transmission of the virus at different stages throughout the rearing of chickens Cook and Darbyshire (1981). McFerran (1979) also reported the lateral spread of the virus because small farmers raise broilers and laying chickens on the same premises. An infected flock of broilers may be a potential risk to laying chickens and hence regular monitoring of broiler flocks for infection should be an essential component in the control of the disease.

Infectious Bronchitis Virus (IBV) is a highly contagious acute viral disease of the upper respiratory tract of chickens, it can also replicate in epithelial tissues of kidneys, gonads and oviduct of chickens causing their pathology and affecting the performance Lee et al. (2004).

Prevention of IB is achieved mainly through vaccination. Although in most cases IBV strains within a geographic region are distinct as mentioned by Callison et al. (2001), Gelb et al. (2005), Ignjatovic et al. (2006). Because IBV undergoes frequent changes in the viral genome, mainly in the S1 gene which result in point mutations promoting the emergence of new antigenic variants Bochkov et al. (2007), Ammayappan et al. (2008) and Lee et al. (2008). The multiple IBV serotypes and its antigenic variation adds complexity to the proper selection of vaccination protocol and proper selection of serologic method to analyses the test results Jackwood and De Wit (2013). Vaccine strains should be selected to represent the antigenic spectrum of isolates in a particular region, because attenuated vaccines are known to have a limited range of protection, confined in many cases to homologous strains, rendering vaccination partially successful Lin et al. (2005). The use of heterologous vaccine strains, either simultaneous or sequentially, has broadened the protection spectrum in some cases Cook et al. (1999), but it is difficult to predict which combinations may confer the best protection. On the other hand, more virulent vaccine strains may have a broader range of protection, but their use is not recommended to avoid the risk of a disease outbreak Darbyshire (1985).

In the present study HI for IBV using the D-274 antigen of the German variant led us to conclude that the majority of the examined flocks were seropositive after neglecting the cutoff values and considering that., these flocks didn't receive such protective vaccines as seen with sample $(2,3,8,10,11,14,17$, $18,19,21,24,26,27$ and 30 ). On the other hand Infection with Mass type IBV was noticed in (sample 8)., the history of this flock shows a drop in egg production and deformed egg quality. This flock was re-examined in (sample 20) at 51 week of age where a high immune titers was still detectable.

Abdelwhab and Abdel-Moneim (2015) had wondered! Will Egypt be the epicenter of the next influenza pandemic? We think yes especially if we consider viral Concurrency as seen in (table 3-4) beside neiglecance in poultry operation.

During investigation of the increased morality or dropped egg production in the examined flocks bacteriological work was performed parallel to the serosurvillance surprisingly a very resistant bacteria was detected, these resistant bacteria was initially identified as klebsilla spp. further examination is ongoing.

\section{REFRANCES}

Abdelwhab, EM.; Veitsa, J. and Mettenleitera, TC. (2013): Genetic changes that accompanied shifts of LPAIV toward higher pathogenicity in poultry. Virulence 4: 441-452.

Abdelwhab, EM.; Veitsa, J. and Mettenleitera, TC. (2014): Prevalence and control of $\mathrm{H}_{7}$ AIV viruses in birds and humans. Epidemiol. Infect. 142: 896-920.

Abdelwhab, EM. and Abdel-Moneim, AS. (2015): Epidemiology, ecology and gene pool of AIV in Egypt: Will Egypt be the epicenter of the next influenza pandemic? Virulence 6: 6-18.

Afifi, M.; Hassan, M.; El-Kady, M.; Zoelfakar, S. and Aly, M. (1999): Prevalence of AI in Egypt. J. of Egypt. Vet. Med. Assoc. 59: 1271-1284.

Afifi, MA.; El-Kady, MF.; Zoelfakar, S. and AbddelMoneim, AS. (2013): Serological surveillance reveals widespread influenza A H7 and H9 subtypes among chicken flocks in Egypt. Trop. Animal Health and Prod.; 45: 687-690.

Ahmed, AAS. and El-Sisi, MA. (1969): CELO virus infection in chickens. Clinical and serologic response to experimental and accidental infection, Avian Dis 13, 709-720.

Alexander, DJ. (1997): ND and other Paramyxoviridae infections. In Diseases of Poultry. $10^{\text {th }}$ ed: Calnek, B.W., Barnes, H.J., Beard, C.W., Mcdougald, L.R., Saif, Y.M. Iowa State University Press, Ames, Iowa, pp. 541-569.

Alexander, DJ.; Bell, JG. and Alders, RG. (2004): Technology Review: Newcastle disease. FAO animal production and health paper 161, Rome, $22 \mathrm{pp}$.

Allan, WH.; Lancaster, JE. and Toth, B. (1978): Newcastle disease vaccines their production and use. FAO animal production and Health series No. 10.

Al-Natour, MQ. and Abo-Shehada, MN. (2005): Seroprevalence of avian influenza among broiler-breeder flocks in Jordan. Preventive Veterinary Medicine, (70), 45-50.

Aly, MM.; Arafa, A.; Kanawaty, Z.; Abdel-Whab, EM. and Hassan, MK. (2008): Avian influenza $\mathrm{H} 7$ surveillance on backyard birds around El- 
Abassa Lake in Egypt, 2007. In: Emerging and Exotic Viral Infection Challenging Threats of Human, Animal and Plant Health, Egyptian Society of Virology, Cairo, Egypt, 5-6 th April.

Aly, MM.; Arafa, A.; Kilany, WH.; Sleim, AA. and Hassan, MK. (2010): Isolation of LPAIV $\left(\mathrm{H}_{7} \mathrm{~N}_{7}\right)$ from a black kite (Milvus migrans) in Egypt in 2005. Avian Diseases, 54, 457-460.

Ammayappan, A.; Upadhyay, C.; Gelb, JJr. and Vakharia, VN. (2008): Complete genomic sequence analysis of IBV Ark DPI strain and its evolution by recombination .Virol J. 5:157.

Awad, FI.; Bassiouni, AA.; El-Sisi, MA. and Youssef, YI. (1973): Clinical and serological response of turkey to artificial infection with AAV type 1 (CELO) Egypt. J. Vet. Sci. 10, 65-74.

Beard, CW. and Hanson, RP. (1981): In Hofstad, M.S. (Ed.), Diseases of Poultry. Iowa State University Press, Ames, pp. 457-470.

Bochkov, YA.; Tosi, G.; Massi, P. and Drygin, VV. (2007): Phylogenetic analysis of partial S1 and $\mathrm{N}$ gene of IBV isolates from Italy revealed genetic diversity and recombination. Virus Genes .35:65-71.

Bradbury, JM. (1984): Avian mycoplasma infections: Prototype of mixed infections with mycoplasmas, bacteria and viruses. Annalesdemicrobiologie 135A (1) 83-89.

Callison, SA.; Jackwood, MW. and Hilt, DA. (2001): Molecular characterization of IBV isolates foreign to the United States and comparison with USA isolates. Avian Dis. 45: 492-499.

Chrysostome, CAAM.; Bell, JG.; Demey, F. and Verhulst, A. (1995): Seroprevalences to three diseases in village chickens in Benin. Prev. Vet. Med. 22, 257-261.

Comin, A.; Toft, N.; Stegman, A.; Klinkenberg, D. and Marangon, S. (2013): Serological diagnosis of AIV in poultry: is the HI test really the 'gold standard'? www.influenza journal.com

Cook, JKA.; Orbell, SJ.; Woods, MA. and Huggins, MB. (1999): Breadth of protection of the respiratory tract provided by different liveattenuated infectious bronchitis vaccines against challenge with infectious bronchitis viruses of heterologous serotypes. Avian Pathol. 28: 477-485.

CooK, JKA. and Darbyshir, JH. (1981): Longitudinal studies on $\left(\mathrm{EDS}_{76}\right)$ in the fowl following experimental infection at 1 day old. Avian Pathology, I0, 449-459.

Darbyshire, JH. (1985): A clearance test to assess protection in chickens vaccinated against avian IBV. Avian Pathol. 14: 497-508.

Dhillon, AS. and Kibenge, FSB. (1987): Adenovirus infection associated with respiratory disease in commercial chickens. Avian Dis. 31: 654-657.
Du Bose, RT.; Grumbles, LC. and Flowers, AI. (1958): The isolation of a non-bacterial agent from quail with a respiratory disease. Poult. Sci. 37, 654-658.

Du Bose, RT. and Gumbles, LC. (1959): The relationship between quail bronchitis virus and chicken embryo lethal orphan virus. Avian Dis. 3, 321-344.

Easterday, BC.; Virginia, SH. and Halvorson, DA. (1997): Influenza: In Diseases of Poultry. $10^{\text {th }}$ ed: Calnek, B.W., Barnes, H.J., Beard, C.W., Mcdougald, L.R., Saif, Y.M. (Eds.),. Iowa State University Press, Ames, Iowa, pp. 583-605.

Eid, MA. (1994): Studies on some avian viruses with special preference to a heat resistant NDV. A thesis submitted to zagazig Univ. for the Ph.D. of Vet. Medical science

El-Zoghby, EF.; Arafa, AS.; Hassan, MK.; Aly, MM.; Selim, A.; Kilany, WH.; Selim, U.; Nasef, S.; Aggor, MG.; Abdelwhab, EM. and Hafez, HM. (2012): Isolation of $\mathrm{H}_{9} \mathrm{~N}_{2}$ avian influenza virus from bobwhite quail (Colinus virginianus) in Egypt. Archives of Virology, 157: 1167-1172.

Fadly, AM. and Winterfield, RW. (1973): Isolation and some characteristics of an agent associated with inclusion body hepatitis, hemorrhages and aplastic anemia in chickens. Avian Dis. 17: 182-193.

Feare, CJ. (2010): Role of wild birds in the spread of HPAIV H5N1 and implications for global surveillance. Avian Diseases 54, 201-212.

Fred leung (2012): (Prof, School of Biolog. Sci., Univ. of Hong Kong.fcleung@hku.hk).

Gelb, JJ.; Weisman, Y.; Ladman, BS. and Meir, R. (2005): S1 gene characteristics and efficacy of vaccination against IBV field isolates from the USA and Israel (1996-2000). Avian Pathol.34:194-203.

Gelb, JJr. (1989): IBV in: a lab. manual of isolation and identification of avian pathogens, 3rd ed. Purchase H.G., L.H. Arp., C.H. Domermth and J.E. Pearson Ed. AAAP., Kennett squar.

Hoffmann, R.; Wessling, E.; Dorn, P. and Dangschat, H. (1975): Lesions in chickens with spontaneous or experimental infectious hepatomyelopoielic disease (inclusion body hepatitis) in Germany. Avian Dis. 19: 224-236.

Ignjatovic, J.; Gould, G. and Sapats, S. (2006): Isolation of a variant IBV in Australia that further illustrates diversity among emerging strains. Arch Virol.151: 1567-1585.

Ismail, A. (1966): Virus isolation aus den caecalen Tonsillen des Huhnes. Ing. Diss, Hannover. Germany.

Jackwood, MW. and De Wit, JJ. (2013): IBV. In: Dis. of Poult. $13^{\text {th }}$ edit. Edit. David E. Swayne pp139.

Kaleta, EF. (1992): Paramyxovirusinfektionen. In: Heider, G., Monreal, G. (Eds.), Krankheiten 
des WirtschaftsgefluÈgels, B and 1. Gustav

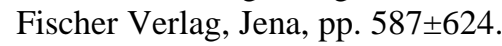

Khafagy, A.; Amin, A. and Abd-El Salam, A. (1995): Serological survey on avian influenza antibody in chicken and turkey flocks. J. of Egyptian Vet. Med. Assoc., 55: 1167-1172.

Khafagy, A.; Sheble, A.; Sarhan, A. and Gough, R. (1992): Isolation of influenza virus (subtype $\mathrm{H}_{7} \mathrm{~N}_{1}$ ). In: Proceeding II, Congress of Facult. of Vet. Med. Cairo Univ., Cairo, Egypt, pp. 65-68.

Lee, CW.; Brown, C.; Hilt, DA. and Jackwood, MW. (2004): Nephropathogenesis of chickens experimentally infected with various strains of IBV. J. Vet. Med. Sci. 66:835-840.

Lee, EK.; Jeon, WJ.; Lee, YJ.; Jeong, OM.; Choi, JG.; Kwon, JH. and Choi, KS. (2008): Genetic diversity of IBV isolates in Korea between 2003 and 2006. Avian Dis. 52: 332-337.

Lin, KY.; Wang, HC. and Wang, CH. (2005): Protective effect of vaccination in chicks with IBV against field virus challenge. J. Microbiol Immunol Infect. 38: 25-30.

McFerran, JB. (1979): Egg drop syndrome. Vet Quart, 1: 176-180.

McFerran, JB. (1981): Adeno viruses of vertebrate animals. In Comparative diagnosis of viral diseases III. E. Kurstak and C. Kurstuk (eds.). pp. 102-165. Academic press, New York.

McFerran, JB. (1989): Adeno viruses in: A laboratory manual for the isolation and identification of avian pathogens. 3rd ed. Purchase H.G., L.H. Arp., CH, Domernuth, and J.E. Pearson Eds. AAAP. Kennett square Pa. pp. 77-81.

McFerran, JB. (1991): AdenoV. in Dis.of poult. $9^{\text {th }}$ ed. B.W. Calnek H. John Barnes, C.W. Beard, W.M. Reid and H.W Yoder Iowa State Univ. Press, Ames. pp. 552-582.

McFerran, JB. and Stuart, JC. (1990): Adenoviruses in : Poultry diseases, $3^{\text {rd }}$ ed. Jordan F.T.W., Bailliere Tindall Loudon N.W.1 7DX; England pp.: 182-193.

Meulemans, G.; Dekegel, D.; Peeters, J.; Van Meirhaeghe, E. and Halen, P. (1979): Isolating adenov. from laying chickens affected by $\left(\mathrm{EDS}_{76}\right)$. Vlaams Diergeneeskd Tijdschr, 2: 151-157.

Monereal, G. (1966): Der Einfluss des CELO-virus auf die kunstliche Infektion mit Mycoplasma gallisepticum. Berl. Munch. TierarztL. Wschr. 79, 295-297.

Monereal, G. (1968): Untersuchungen uber ein aviares Adeno virus Habi1_ Schrift. Zbl. Vet. Med. B 10, 607-614.

Monereal, G. and Ahmed, AAS. (1963): CELO-V als komplizie render faktor beivirologischen Untersuchungen von Atems organen der Huneher Zb1. Vet. Med. B 10, 607-614.

Morrow, CJ. (2008): Laboratory Investigations to support health programmes and disease diagnosis .in: Poultry Diseases, edited by Mark Pattison, Paul F McMullin, Janet M. Bradbury, Dennis J Alexander, chapter; Elsevier Health Sciences.

Mousa, S.; Bayoumi, A.; Gad, N. and El-Demerdach, $M$. (1984): Inclusion body hepatitis complex in chicken at Elwaady-E1 Gadeed Egypt. Assiut Vet. Med. J. 13: 83-94.

Nawathe, DR. and Abegunde, A. (1980): $\mathrm{EDS}_{76}$ in Nigeria: Serological evidence in commercial farms. Vet. Rec., 107: 466-467.

OIE (2010): AIV. In Manual of Diagnostic Tests and Vaccines for Terrestrial Animals OIE, Paris.

Olson, NO. (1950): A respiratory disease (bronchitis) of quail caused by a virus. Proc. 54th Annual Meet. US Livestock Sanit Assoc, pp: 171-174.

Osbaldiston, GW. (1973): Laboratory procedures in clinical vet. Bacteriologies. Yale University. School of medicine university park, Press Baltimore - London - Tokyo international publishers in science and medicine chamber of commerce building. Balitmore, Maryland 21202.

Papanikoloau, I.; Iordanidis, P. and Artopios, E. (1985): Mixed infection with adenovirus and IBV in two flocks of brown layers. Ellenike kteniatrike- Hellenic- Veterinary- Med. 28: 209-216.

Rosenberger, JK.; Eckroade, RJ.; Klopp, S. and Krauss, WC. (1974): Characterization of several viruses isolated from chickens with inclusion body hepatitis and aplastic anemia virus in broilers Trop. Ani. Hlth prod. 27: 167170.

Singh, JK.; Singh, KCP.; Prasad, CB. and Prasad, C. (1995): Occurrence of ${ }^{\prime} \mathrm{HI}$ antibodies against egg drop syndrome 1976 virus in broilers. Trop. Anim. Hlth Prod. 27, 167-170.

Snedecor, GW. and Cochran, WG. (1967): Statistical methods, $6^{\mathrm{TH}}$ ed., Univ press, Ames., Iowa. USA.

Soliman, A.; Saad, M.; Elassal, E.; Amir, E.; Plathonoff, C.; Bahgat, V.; El-Badry, M.; Ahmed, LS.; Fouda, M.; Gamaleldin, M.; Mohamed, NA.; Salyer, S.; Cornelius, C. and Barthel, R. (2012): Surveillance of avian influenza viruses in migratory birds in Egypt, 2003-09. J. of Wild Life Dis. 48: 669-675.

Timm Harder (2012): Friedrich-Loeffler-Institut Bundesforschungsinstitut für Tiergesundheit Federal Research Institute for Animal Health Südufer 10 | 17493 Greifswald - Insel Riems Tel: +49 383517 1546/1152 | Fax: +49 38351 71174 http://www.fli.bund.de/

VAN Ecz, JHH. (1982): Serological examination and egg production of progeny of fowl experimentally infected with "E D S 76 " virus. Veterinary Quarterly, 4:117-124. 
Villegas, PC. (2006): Lab. manual .avian virus diseases. pp 33, 37-38. Coll. Vet. Med. Athens, Georgia.

Winterfield, $R W$. (1984): Avian adenovirus infections in: Diseases of poultry, ed. Hofstad M.S.
Yates, VJ.; YO; Rhee, DE.; Fry, AM.; El Mishad, KJ. and McCormick (1976): The presence of avian adenoviruses and adenovirus associated viruses in healthy chickens. Avian Dis. 20: 146-152.

\title{
دلالات وبائية عن بعض المسبيات الفيروسية المصاحبة لارتفاع النفوق في الاجاج المربى بمحافظة الثرقية
}

\author{
هشام احمد عبل البلبيع محمد ، علا عادل حسنبن \\ Email: heshambayoumie@yahoo.com \\ Assiut University Email: www.aun.edu.eg
}

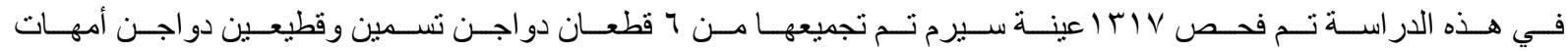

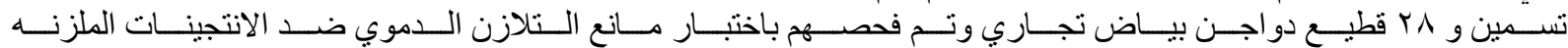

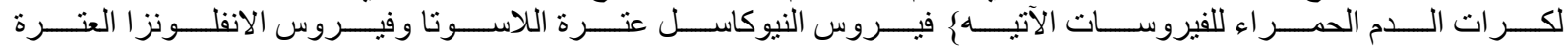

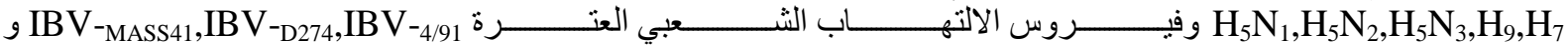

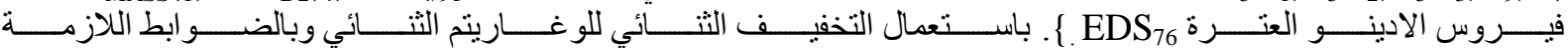

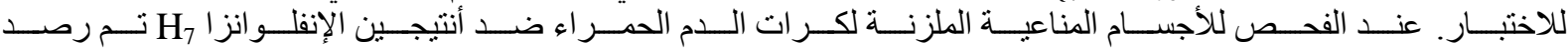

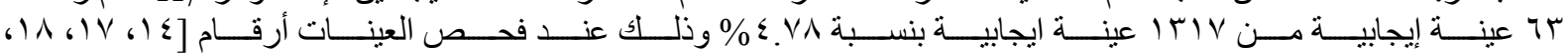

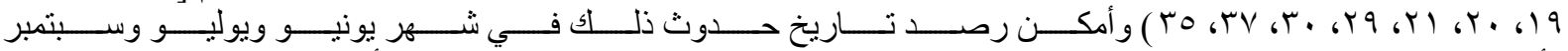

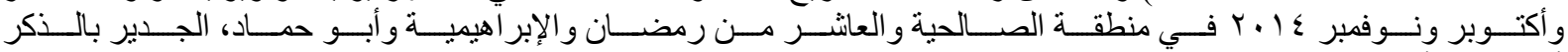

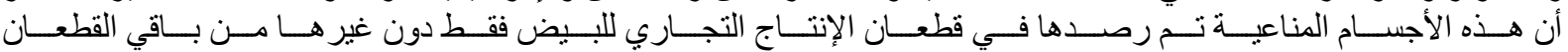

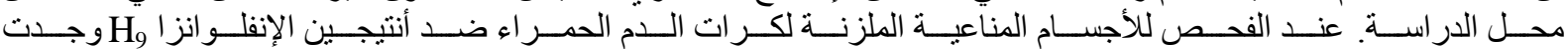

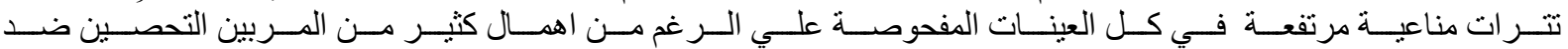

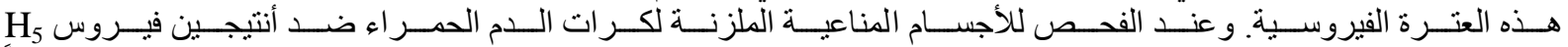

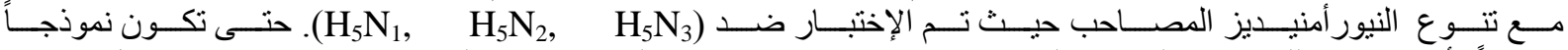

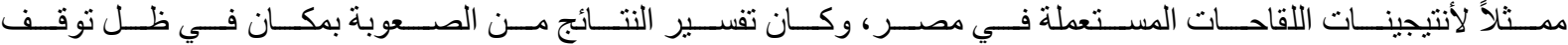

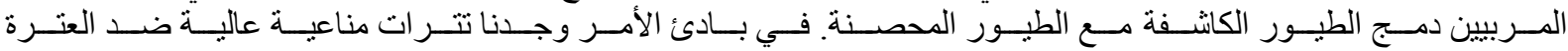

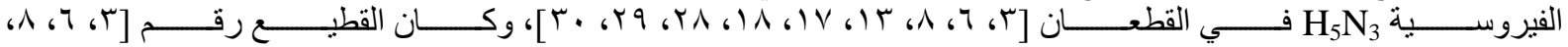

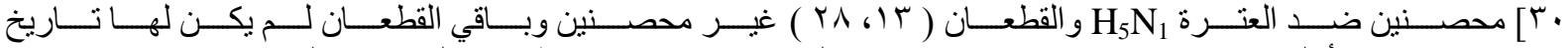

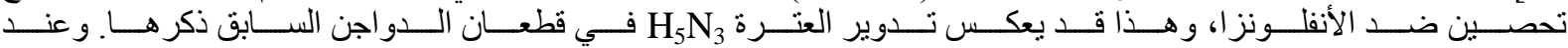

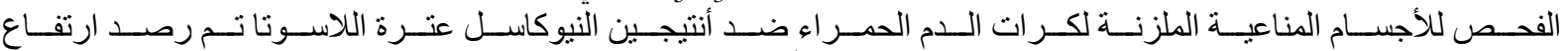

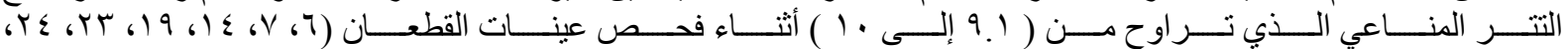

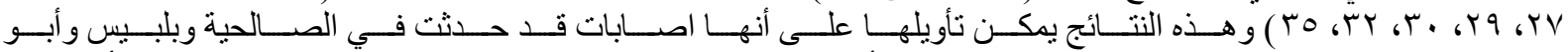

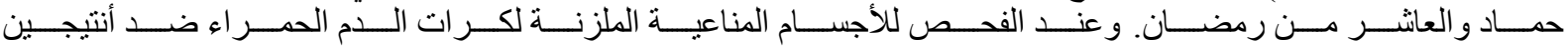

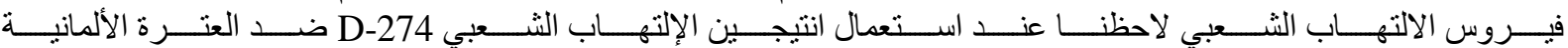

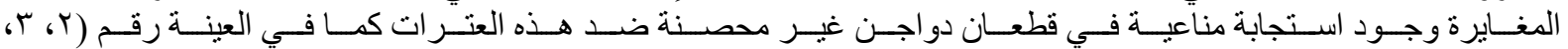

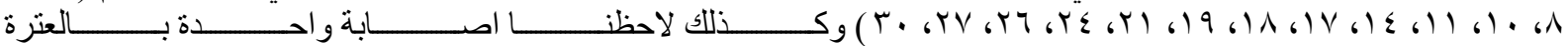

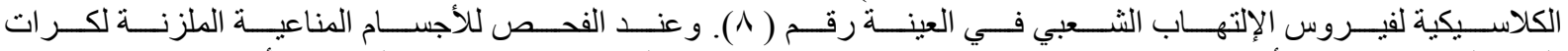

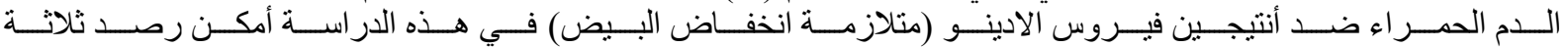

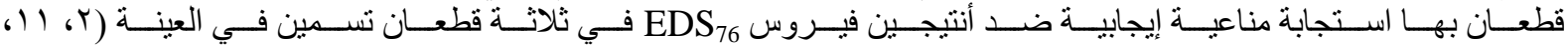

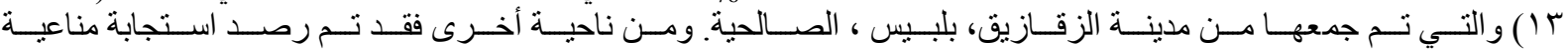

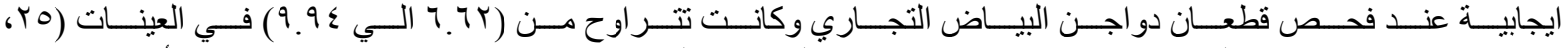

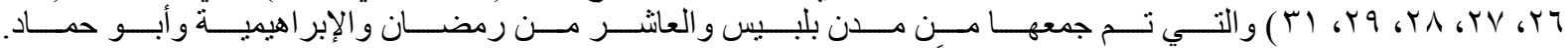

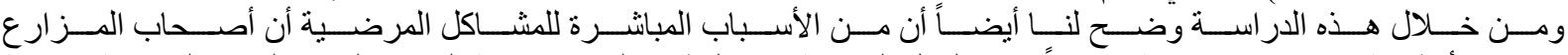

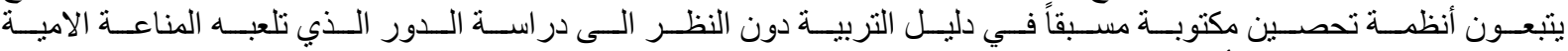

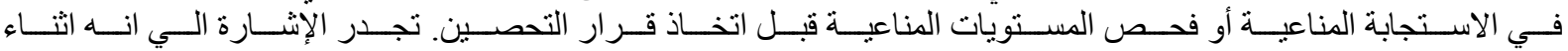

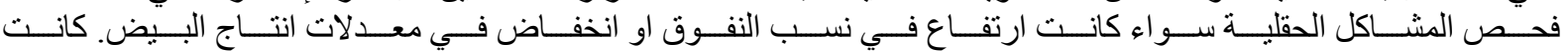

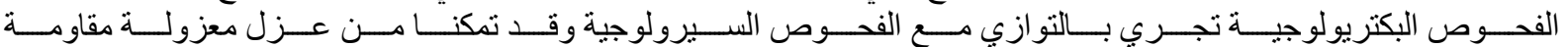

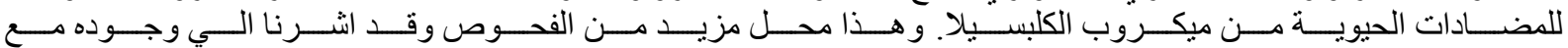
التداخلات الفيروسية التي اشرنا اليها لمحاولة تبرير مسببات النفوق المرتفع في محافظة الثرقية. 